\title{
Semi-Abelian Schemes and Heights of Cycles in Moduli Spaces of Abelian Varieties
}

\author{
Jean-Benoît Bost (*) - Gerard Freixas i Montplet (**) \\ Questo articolo è dedicato a Francesco Baldassarri \\ con i nostri migliori auguri
}

ABSTRACT - We study extension properties of Barsotti-Tate groups and we establish diophantine inequalities involving heights of cycles with respect to logarithmically singular hermitian line bundles on arithmetic varieties. We apply these results to bound heights of cycles on moduli spaces of abelian varieties, induced by quotients of abelian varieties by levels of Barsotti-Tate subgroups, over function fields over number fields. To achieve this aim, we combine our results with an effective version of Rumely's theorem on integral points on possibly open arithmetic surfaces and with Faltings' theorems on heights of abelian varieties in isogeny classes.

\section{Introduction}

Let $k$ be a field and $A$ a $g$-dimensional abelian variety over $k$. For any prime number $l$ different from the characteristic of $k$, the $l$-adic Tate module is defined as

$$
T_{l} A=\lim _{\overleftarrow{n}} A\left[l^{n}\right](\bar{k})
$$

where $A[n]$ denotes the $n$-torsion subgroup scheme of $A$ and $\bar{k}$ an algebraic closure of $k$. It is well known that $T_{l} A$ is a free $Z_{l}$-module of rank $2 g$. The action of the absolute Galois group $G_{k}:=\operatorname{Gal}(\bar{k} / k)$ of $k$ on the $\bar{k}$ -

(*) Indirizzo dell'A.: Département de Mathématiques, Université Paris-Sud 11, Bâtiment 425, 91405 Orsay cedex, France.

E-mail: jean-benoit.bost@math.u-psud.fr

(**) Indirizzo dell'A.: CNRS-Institut de Mathématiques de Jussieu, 4 Place Jussieu, 75005 Paris, France.

E-mail: freixas@math.jussieu.fr 
valued torsion points of $A$ endows $T_{l} A$ with the structure of a Galois module. Besides, the endomorphism ring $\operatorname{End}_{k}(A)$ (consisting of the $k$ endomorphisms of the abelian variety) is a torsion free Z-algebra of finite type. The Q-algebra $\operatorname{End}_{k}(A) \otimes_{\mathrm{Z}} \mathrm{Q}$ is semi-simple (and consequently, for any $l$ as above, the $Q_{l}$-algebra $\operatorname{End}_{k}(A) \otimes_{\mathrm{Z}} \mathrm{Q}_{l}$ also), and the action of $\operatorname{End}_{k}(A)$ on $T_{l} A$ is compatible with the structure of $G_{k}$-module on $T_{l} A$.

THEOREm 1.1 (Tate's conjecture for abelian varieties). Assume that $k$ is finitely generated over its prime field. The following assertions hold:

a. $T_{l} A \otimes_{\mathrm{Z}_{l}} \mathrm{Q}_{l}$ is a semi-simple $\mathrm{Q}_{l}\left[G_{k}\right]-$-module;

b. the natural map

$$
\operatorname{End}_{k}(A) \otimes_{\mathrm{Z}} Z_{l} \longrightarrow \operatorname{End}_{Z_{l}\left[G_{k}\right]}\left(T_{l} A\right)
$$

is an isomorphism.

Theorem 1.1 was proven by Tate himself when $k$ is a finite field [32], and by Zarhin when $k$ is a function field over a finite field [34, 35] (see also [22], notably Chapter XII). Faltings established it in the number field case in his famous article [12] (see also [13], [15], [30]). His result was extended to the case of a field $k$ finitely generated over Q in [15, Chap. VI] and [30, Exp. IX].

In all cases, the proof is based on the following proposition:

Proposition 1.2 (Tate). Let A be an abelian variety over a field $k$. Let $l$ be a prime number different from the characteristic of $k$. Suppose that $A$ satisfies the following property:

$\mathcal{T}(A / k)$ : for any l-divisible group $\left\{G_{n}\right\}_{n \geq 0}$ with $G_{n} \subseteq A\left[l^{n}\right]$, infinitely many of the abelian varieties $A / G_{n}$ over $k$ are isomorphic to each other.

Then

i. for every $Q_{l}\left[G_{k}\right]$-submodule $W$ of $T_{l} A \otimes_{\mathrm{Z}_{l}} \mathrm{Q}_{l}$, there exists $u$ in $\operatorname{End}_{k}(A) \otimes_{\mathrm{Z}} \mathrm{Q}_{l}$ such that $u\left(T_{l} A \otimes_{\mathrm{Z}_{l}} \mathrm{Q}_{l}\right)=W$;

ii. Theorem 1.1 holds for the abelian variety $A$.

Proofs of $\mathcal{T}(A / k)$ have to deal with the difficulty of establishing finiteness results for isomorphism classes of abelian varieties not endowed with a polarization. A crucial contribution of Zarhin's [35] has been to introduce the so-called "Zarhin trick", that allowed him to get rid of the (lack of) polarization problems, and to establish the following result: 
Proposition 1.3 (Zarhin). Let $k$ be a field, and $l$ be a prime number different from the characteristic of $k$.

Assume that, for every abelian variety $A$ over $k$ and any principal polarization $\lambda$ over $A$ (defined over $k^{1}$ ), the following property is satisfied:

$\mathcal{Z}(A, \lambda / k)$ : for any l-divisible group $\left\{G_{n}\right\}_{n>0}$ with $G_{n}$ a subgroup of $A\left[l^{n}\right]$ lagrangian with respect to the Weil pairing attached to $\lambda$, the quotients $A / G_{n}$ belong to a finite family of $k$-isomorphism classes.

Then Property $\mathcal{T}(A / k)$ and Theorem 1.1 hold for any abelian variety $A$ over $k$.

We refer to [32] and to [15, Chap. IV, Sec. 2] for discussions of the reduction of the Tate conjecture to assertions $\mathcal{T}(A / k)$ and $\mathcal{Z}(A, \lambda / k)$.

The method of Faltings for establishing $\mathcal{Z}(A, \lambda / k)$ when $k$ is a number field combines results of Tate and Raynaud on Barsotti-Tate groups, and some ideas from Arakelov geometry. If indeed $K$ denotes a number field and $\mathcal{O}_{K}$ its ring of integers, Faltings attaches some canonical height $h_{F}(A)$ to (the Néron model over $\mathcal{O}_{K}$ of) an abelian variety $A$ over $K$. The Faltings height is a counterpart of Zarhin's geometric height used in [34], and a key point in Faltings proof is a finiteness property $\grave{a}$ la Northeott for this height [12, Sec. 3].

When $k$ is finitely generated over $\mathrm{Q}$, the proof of $\mathcal{Z}(A, \lambda / k)$ and of Theorem 1.1 in [15, Chap. VI] and [30, Exp. IX] uses - besides Faltings original arguments in the number field case - some results of Deligne or Faltings concerning abelian schemes over varieties on fields of characteristic zero ([8], [11]) that are established by Hodge theoretic methods.

It is possible to avoid the use of Hodge theory in the proof of $\mathcal{Z}(A, \lambda / k)$ when $k$ is finitely generated over some field $k_{0}$ by using Zarhin's results on bounded families of abelian schemes over (open dense subschemes) of a normal projective scheme $X$ over $k_{0}$ (here $k_{0}=\mathrm{Q}$, and $k_{0}(X)=k$ ) in their full strength version established by Moret-Bailly [22, Chap. XII] to show that, with the notation used in $\mathcal{Z}(A, \lambda / k)$ above, the quotients $A / G_{n}$ arise in some bounded family. By considering the restrictions of these quotients to suitable many closed points of $X$, and by using the validity of $\mathcal{Z}(A, \lambda / k)$ when $k$ is a number field, one concludes to the finiteness of their isomorphism classes.

(1) Namely, such that the attached isogeny $A \rightarrow A^{\vee}$ from $A$ to its dual abelian variety is defined over $k$. 
In this note we sketch a variant to these diverse approaches to $\mathcal{Z}(A, \lambda / k)$, in the situation where $k$ is a function field of transcendence degree 1 over Q. Our approach also avoids Hodge theoretic arguments, but relies on higher-dimensional Arakelov geometry. It involves bounds on heights of cycles in relevant moduli spaces, and consequently provides some kind of "quantitative control" in the finiteness property asserted by $\mathcal{Z}(A, \lambda / k)$ or $\mathcal{T}(A / k)$, in the spirit of [30, Exp. VII, 4.4].

Actually our derivation of $\mathcal{Z}(A, \lambda / k)$ provides us with an excuse to deal with the following subjects, which constitute the core of the article:

$i$. extension properties of Barsotti-Tate subgroups of abelian schemes with semi-stable reduction, over two dimensional regular bases (section 2);

ii. heights of cycles on arithmetic varieties, with respect to logarithmically singular hermitian line bundles (section 3). These heights satisfy a Liouville type inequality (Theorem 3.2) and Northcott's finiteness property (Corollary 3.4);

iii. a generalization to logarithmically singular hermitian line bundles of a theorem of Zhang for heights of generic sequences of points in arithmetic varieties (Theorem 3.5);

$i v$. a theorem of Rumely [29], and a refinement by Autissier [2], on the existence of integral points on open arithmetic surfaces. This is combined with the previous points and Faltings' theorem [12] on heights of quotients of an abelian scheme by the levels of a BarsottiTate subgroup (section 4).

Most of our constructions might be extended to function fields of arbitrary positive transcendence degree over Q. For the sake of simplicity, we have considered the case of transcendence degree 1 only. Observe also that, by using the work of Zarhin and Moret-Bailly as discussed above, the validity of $\mathcal{Z}(A, \lambda / k)$ when $k$ is a function field of transcendence degree larger than 1 over $\mathrm{Q}$ may be derived from its validity when $k$ has transcendence degree 1 , combined with purely geometric arguments.

To our knowledge, the considerations in $i-i i i$, although not unexpected, do not appear in the literature. The use made of them in $i v$ shows that they may be of interest for diophantine geometry. More than the application to the Tate conjecture over function fields, we believe that the interest of this text lies in the tools we develop. We expect them to be useful in other contexts. Finally, let us mention the related work of H. Ikoma [19], that also avoids the use of complex Hodge theory by using instead the theory of heights developed by Moriwaki [24]. 


\section{Barsotti-Tate subgroups of semi-abelian schemes}

2.1. Let $S$ denote a regular, integral and noetherian scheme of Krull dimension 2 . We denote its generic point by $\eta$ and suppose its characteristic to be 0 . In the sequel, we fix a prime $p$ and we deal with Barsotti-Tate subgroups in the $p$-torsion of abelian schemes. Only basic notions concerning Barsotti-Tate groups will be required. To shorten the exposition, we refer the reader to Tate's article [33] and Illusie's exposé [30, Exp. VI] for an introduction.

Proposition 2.1. Let to be a semi-abelian scheme over $S$ with abelian generic fiber $A$, and $\left\{G_{n}\right\}_{n \geq 0}$ a Barsotti-Tate subgroup of $A\left[p^{\infty}\right]$. Assume that, for every $n \geq 0$, there exists a finite flat group subscheme $\mathscr{P}_{n} \subset$ to such that $G_{n} \subset \mathscr{P}_{n, \eta}$. Then there exists a big open subset $U$ of $S$ (namely an open subset $U$ such that $\operatorname{codim}(S \backslash U) \geq 2$ ) and an integer $m \geq 0$ such that:

$i$. $G_{m}$ extends to a finite flat group subscheme $\mathscr{G}_{m} \subset \mathscr{A}\left[p^{m}\right]_{\mid U}$;

ii. the Barsotti-Tate group defined by $H_{n}=G_{m+n} / G_{m}$ extends to a Barsotti-Tate group $\left\{\mathscr{H}_{n}\right\}_{n>0}$ over $U$;

iii. let $\mathscr{B}=\mathscr{C}_{\mid U} / \mathscr{G}_{m} \cdot{ }^{2}$ Then $\mathscr{H}_{n}$ is a group subscheme of $\mathscr{B}\left[p^{n}\right]$ for all $n \geq 0$.

REMARK 2.2. $i$. Let $m \geq 0$ be such that the assertion of the proposition holds. We claim that for any $m^{\prime} \geq m$, the analogous assertion obtained by replacing $m$ by $m^{\prime}$ is also true. For this, it is enough to show that $G_{m+n}$ extends to a finite flat group subscheme of $\mathscr{b}_{\mid U}$, for every $n \geq 0$. Define $\mathscr{G}_{m+n}$ to be the kernel of the isogeny

$$
\mathscr{C}_{\mid U} \rightarrow \mathscr{B} \rightarrow \mathscr{B} / \mathscr{H}_{n} .
$$

It is a quasi-finite flat subgroup of $\mathscr{C}_{\mid U}$ containing $\mathscr{G}_{m}$ and such that the quotient $\mathscr{G}_{m+n} / \mathscr{G}_{m}$ is isomorphic to $\mathscr{H}_{n}$. Because $\mathscr{G}_{m}$ and $\mathscr{H}_{n}$ are finite over $S$, we infer that $\mathscr{G}_{m+n}$ is finite as well.

$i i$. After possibly replacing $U$ by a smaller big open subset, one can suppose that all the groups $G_{n}$ extend to finite flat subgroups of $\mathscr{C}_{\mid U}$.

$\left(^{2}\right)$ The quotient is well defined because $S$ is regular and $\mathscr{b}$ is generically abelian. This ensures the existence of relatively ample line bundle $\mathscr{A} / S[27$, Cor. XI 1.16], and hence the quotient by a finite flat subgroup is defined [SGA III, 5.4.1]. 
Indeed, we already saw that this is true for all $n \geq m$. On the other hand, the finitely many remaining groups $G_{n}, n \leq m$, extend to finite flat subgroups of $\mathscr{C}_{\mid U^{\prime}}$, for some other big open subset $U^{\prime}$. For this, recall that $G_{n} \subseteq \mathscr{P}_{n, \eta}$ and $\mathscr{P}_{n}$ is a finite flat subgroup of $\mathscr{C}$. Thus, replacing $U$ by $U \cap U^{\prime}$, we obtain a big open subset for which the claim holds.

The purity lemma [14, Chap. V, Lemma 6.2] will be needed in the course of the proof of Proposition 2.1. We quote it for the convenience of the reader.

LEMma 2.3. Let $R$ be a regular local ring of Krull dimension 2. Write $S=\operatorname{Spec} R, \eta$ for the generic point of $S, s$ for the closed point of $S$, $U=S \backslash\{s\}$ and $j: U \hookrightarrow S$ for the open immersion. The functor $j_{*}$ defines an equivalence between the categories of locally free sheaves of finite rank over $U$ and over $S$. Moreover it is compatible with tensor product.

REMARK 2.4. It is important to bring the reader's attention to the following subtlety: the functor $j_{*}$ does not preserve exact sequences. Actually, de Jong-Oort show in [18, Sec. 6] that it is possible to find a regular local scheme $S$ of dimension 2 and generic characteristic 0, finite flat group schemes $G, H$ over $S$ and a morphism $\varphi: G \rightarrow H$ which is a closed immersion over $U=S \backslash\{s\}$ but not over $S$. In particular, the schematic closure of $G_{\eta}$ in $H$ is not flat over $S$.

Proof of Proposition 2.1. By Remark 2.2 and by the noetherianity hypothesis, we may assume that $S=\operatorname{Spec} R$ is affine. The proof is then divided into several steps.

STEP 1. We observe that the group scheme $G_{n}$ can be extended to a finite flat group scheme $\mathscr{G}_{n}$ over $S$, and that there exists a big open subset $U_{n}$ of $S$ such that $\mathscr{G}_{n \mid U_{n}}$ is a subgroup scheme of $\mathscr{C}_{\mid U_{n}}$. This is easily obtained from finite flat closures along codimension 1 closed subsets and Lemma 2.3.

STEP 2. The next point is an adaptation of Tate's trick ([33], proof of Proposition 12 and Lemma 2.5 below) to construct a candidate to the desired Barsotti-Tate group from suitable quotients of the group schemes $\mathscr{G}_{n}$.

Let $k, n \geq 0$ be integers. The intersection $V=U_{k} \cap U_{k+n}$ is a big open subset of $S$. We have inclusions

$$
\mathscr{G}_{k \mid V} \subseteq \mathscr{G}_{k+n \mid V} \subseteq \mathscr{C}_{\mid V}
$$


so there is a well-defined quotient $\mathscr{G}_{k+n \mid V} / \mathscr{G}_{k \mid V}$. This is a finite flat group scheme over $V$. By Lemma 2.3 this group scheme uniquely extends to a finite flat group scheme over $S$, to be denoted $\mathscr{H}_{k}^{k+n}$. If now $V$ denotes another small enough big open subset, then we have a map between well defined quotients

$$
\frac{\mathscr{S}_{k+n+1 \mid V}}{\mathscr{G}_{k+1 \mid V}} \stackrel{p}{\longrightarrow} \frac{\mathscr{S}_{k+n \mid V}}{\mathscr{G}_{k \mid V}}
$$

induced by multiplication by $p$, which is an isomorphism over the generic point. By the purity lemma this morphism extends in a unique way to a morphism of group schemes

$$
\mathscr{H}_{k+1}^{k+n+1} \stackrel{\tilde{p}}{\longrightarrow} \mathscr{H}_{k}^{k+n}
$$

Remark that $\tilde{p}$ is surjective. Indeed $\tilde{p}$ is clearly finite and is dominant too, because the involved groups are flat over $S$ with reduced generic fiber (recall that the characteristic of $\eta$ is 0, so Cartier's theorem applies to the generic fibers, showing they are reduced). Furthermore, $\tilde{p}$ is an isomorphism over the generic fiber. The conditions of Lemma 2.5 below (Tate's trick) are fulfilled: it follows that there exists an integer $m \geq 1$ such that the morphism

$$
\mathscr{H}_{k+1}^{k+2} \stackrel{\tilde{p}}{\longrightarrow} \mathscr{H}_{k}^{k+1}
$$

is an isomorphism for $k \geq m$. Therefore, for every $n \geq 0$, the $n$-th composition

$$
\mathscr{H}_{m+n}^{m+n+1} \stackrel{\tilde{p}^{n}}{\longrightarrow} \mathscr{H}_{m}^{m+1}
$$

is an isomorphism as well.

Define the finite flat group scheme over $S$

$$
\mathscr{H}_{n}=\mathscr{H}_{m}^{m+n} \text {. }
$$

The natural inclusions among the groups $\mathscr{H}_{n}$ restricted on suitable big open subsets extend to a compatible system of morphisms

$$
l_{n}^{n+k}: \mathscr{H}_{n} \longrightarrow \mathscr{H}_{n+k} \text {. }
$$

Let us fix an integer $n \geq 0$. For some big open subset $V$ of $S$ (depending on $n$ ) we have a chain of inclusions

$$
\left.\left.\left.\mathscr{G}_{1}\right|_{V} \subseteq \ldots \subseteq \mathscr{G}_{m+n+1}\right|_{V} \subseteq \mathscr{G}\right|_{V} .
$$


The following diagram commutes:

$$
\begin{gathered}
\mathscr{H}_{n+1 \mid V}=\frac{\mathscr{G}_{m+n+1 \mid V}}{\mathscr{G}_{m \mid V}} \stackrel{p^{n}}{\longrightarrow} \frac{\mathscr{G}_{m+n+1 \mid V}}{\mathscr{G}_{m \mid V}}=\mathscr{H}_{n+1 \mid V} \\
\downarrow^{\pi} \\
\mathscr{H}_{m+n \mid V}^{m+n+1}=\frac{\mathscr{G}_{m+n+1 \mid V}}{\mathscr{G}_{m+n \mid V}^{n+1}} \stackrel{\tilde{p}^{n}}{\longrightarrow} \frac{\mathscr{G}_{m+1 \mid V}}{\mathscr{G}_{m \mid V}}=\mathscr{H}_{1 \mid V} .
\end{gathered}
$$

The morphism $\pi$ is the natural projection. It follows from the diagram that the kernel of multiplication by $p^{n}$ on $\mathscr{H}_{n+1 \mid V}$ is the same as the kernel of $\pi$, namely $\mathscr{H}_{n \mid V}$. Furthermore we have a closed immersion induced by $p^{n}$

$$
\mu_{V}: \frac{\mathscr{H}_{n+1 \mid V}}{\mathscr{H}_{n \mid V}} \hookrightarrow \mathscr{H}_{1 \mid V}
$$

We actually see that $\mu_{V}$ is an isomorphism. Indeed, $\mu_{V}$ is an isomorphism on the generic point $\eta$, so $\mu_{V}$ is dominant. Let $W$ be a big open subset such that $l_{n}^{n+1}: \mathscr{H}_{n \mid W} \rightarrow \mathscr{H}_{n+1 \mid W}$ is a closed immersion. Up to shrinking $V$ we may assume that $V \subseteq W$. Then the isomorphism $\mu_{V}$ extends to an analogous isomorphism $\mu_{W}$, in a unique way, by the purity Lemma 2.3.

SteP 3. We show that $\mathscr{G}_{m}$ and $\left\{\mathscr{G}_{n}\right\}_{n \geq 0}$ are the desired group schemes, after restriction to a suitable big open subset of $S$.

Let $U \subseteq S$ be a big open subset such that $\mathscr{G}_{\left.m\right|_{U}} \subseteq \mathscr{C}_{\mid U}$. To simplify the notations, let us replace $S$ by $U$. There is a well defined quotient semiabelian scheme $\mathscr{B}=\mathscr{b} / \mathscr{G}_{m}$ over $S$. By definition, for every $n$, over some big open subset $V$ we have $\mathscr{H}_{n \mid V} \subseteq \mathscr{P}_{m+n \mid V} / \mathscr{S}_{m \mid V}$. This inclusion uniquely extends to a morphism

$$
\mathscr{H}_{n} \stackrel{h_{n}}{\longrightarrow} \frac{\mathscr{P}_{m+n}}{\mathscr{O}_{m}} \subset \mathscr{B},
$$

by the purity Lemma 2.3. Moreover, the relation $h_{n}=h_{n+1} \circ l_{n}^{n+1}$ holds. In particular, if $h_{n}$ is a closed immersion over a big open subset $W_{n}$, then $l_{n}^{n+1}$ is a closed immersion over $W_{n}$ as well.

Let us now choose a big open subset $W_{n}$ such that $h_{1}$ and $h_{n}$ are closed immersions when restricted to $W_{n}$. We claim that $h_{n+1 \mid W_{n}}$ is a closed immersion as well. Indeed we have a commutative square 


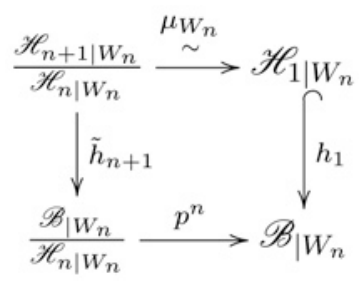

It follows that the arrow $\tilde{h}_{n+1}$ is a closed immersion. We thus have a well defined quotient semi-abelian scheme

$$
\mathscr{C}_{n}=\frac{\mathscr{B}_{\mid W_{n}} / \mathscr{H}_{n \mid W_{n}}}{\operatorname{Im} \tilde{h}_{n+1}}
$$

and a natural isogeny

$$
q: \mathscr{B}_{\mid W_{n}} \longrightarrow \mathscr{C}_{n}
$$

By construction and uniqueness of extensions over $S$, the kernel of the isogeny $q$ is a finite flat group subscheme of $\mathscr{B}_{\mid W_{n}}$ isomorphic to $\mathscr{H}_{n+1 \mid W_{n}}$, and the morphism $h_{n+1}$ is a closed immersion over $W_{n}$.

Let us finally fix a big open subset $U$ of $S$ such that $h_{1}$ is a closed immersion over $U$. Then the preceding argument shows that $h_{n}$ is a closed immersion for all $n$. Furthermore we have isomorphisms

$$
\mu_{U}: \frac{\mathscr{H}_{n+1 \mid U}}{\mathscr{H}_{n \mid U}} \stackrel{\sim}{\longrightarrow} \mathscr{H}_{1 \mid U}
$$

induced by multiplication by $p^{n}$. We conclude that the family $\left\{\mathscr{H}_{n \mid U}\right\}_{n}$ constitutes a Barsotti-Tate group contained in $\mathscr{B} \mid U$. This completes the proof.

LEMMA 2.5 (Tate's trick). Let $S$ be a normal, integral and noetherian affine scheme $S=\operatorname{Spec} R$, of generic characteristic 0 . Let $\left\{X_{k}\right\}_{k>1}$ be a family of finite flat schemes over $S$, with reduced generic fibers, endowed with surjective morphisms $\pi_{k}: X_{k+1} \rightarrow X_{k}$, that become isomorphisms over the generic point of $S$. Then there exists an integer $m \geq 1$ such that $\pi_{k}$ is an isomorphism for every $k \geq m$.

Proof. First of all, we may assume that the schemes $X_{k}$ are nonempty. Indeed, in the empty case the conclusion trivially holds. Let $D_{k} \neq 0$ be the affine $R$-algebra of $X_{k}$. If $F$ is the fraction field of $R$, all the $F$-algebras $D_{k} \otimes_{R} F$ can be identified to a fixed finite $F$-algebra $D$, for instance $D_{1} \otimes_{R} F$. This is so because $\pi_{k}$ is an isomorphism over the generic point $\eta$ for 
all $k \geq 1$. Observe that $D$ is a separable $F$-algebra, because it is reduced by assumption and $F$ has characteristic 0 . The maps $\pi_{k}$ are surjective and the schemes $X_{k}$ are flat, so the $D_{k}$ form an increasing sequence of rings. The $R$ algebra $D_{k}$ contains $R$ as a subring, since $X_{k}$ is flat over $S$ and non-empty. By definition $F \cdot D_{k}=D$. The finiteness condition says that $D_{k}$ is integral over $R$. Hence the ring

$$
\Gamma=\bigcup_{k \geq 1} D_{k}
$$

is an integral extension of $R$ with $F \cdot \Gamma=D$. Therefore, $\Gamma$ is contained in the integral closure of $R$ in the separable $F$-algebra $D$. Since $R$ is noetherian and integrally closed, it follows that $\Gamma$ is a noetherian $R$-submodule of $D$. Consequently, the increasing sequence $D_{k}$ of subrings of $\Gamma$ stabilizes. Let $m \geq 0$ be an integer such that $D_{k}=D_{k+1}$ for all $k \geq m$. Then $\pi_{k}$ is an isomorphism for $k \geq m$.

REMARK 2.6. $i$. The assumption that the schemes $X_{k}$ are reduced cannot be dropped in the preceding statement. Otherwise, one can construct a counterexample. For instance, let us consider a strictly increasing infinite sequence of free $R$-modules of finite constant rank $r$,

$$
E_{1} \subsetneq E_{2} \subsetneq \ldots
$$

Define finite $R$-algebras $D_{k}$ by

$$
D_{k}=R \oplus E_{k}, \quad \text { with } x^{2}=0 \text { for } x \in E_{k} .
$$

The schemes $X_{k}=\operatorname{Spec} D_{k}$ are not reduced but satisfy all the other hypotheses of the lemma. By construction, the conclusion of the lemma is not satisfied: the sequence $\left\{D_{k}\right\}_{k}$ does not stabilize.

ii. As we already recalled in the proof of Proposition 2.1, when the schemes $X_{k}$ are finite flat group schemes over $S$, they are automatically reduced over the generic fiber. This is the case since the generic characteristic of $S$ is 0 , and due to Cartier's theorem: a finite group scheme over a field of characteristic 0 is always reduced. Therefore, in this situation Lemma 2.5 applies.

2.2. We now discuss an application of Proposition 2.1 to the following particular setting. Let $S$ be a local, henselian and integral scheme of dimension 2, whose closed and generic points we denote $y$ and $\eta$ respectively. Let $U=S \backslash\{y\}$ be the unique strict big open subset of $S$. Consider a semiabelian scheme $\mathscr{b} \rightarrow S$ with abelian generic fiber $A$. We can decompose 
the quasi-finite flat groups $\mathscr{A}\left[p^{n}\right]$ over $S$ as

$$
\left.\mathscr{A}\left[p^{n}\right]=\widetilde{\mathscr{A}\left[p^{n}\right.}\right] \cup L_{n},
$$

where $\widetilde{\mathscr{A}\left[p^{n}\right]}$ is the maximal finite subgroup and $L_{n}$ has an empty special fiber [22, Chap. IV, Sec. 1]. These are open and closed flat subschemes of $\mathscr{A}\left[p^{n}\right]$. Moreover this construction is functorial in $S$, with respect to morphisms of local henselian schemes.

Suppose in addition that $S$ is noetherian, complete (hence excellent), and regular (hence normal), of generic characteristic 0 , and that a BarsottiTate subgroup $\left\{G_{n}\right\}_{n}$ of $A\left[p^{\infty}\right]$ is given. The groups $G_{n}$ extend to quasifinite flat closed subgroups $\mathscr{I}_{n} \subset \mathscr{C}\left[p^{n}\right]_{\mid U}$ over $U$, with inclusions $\mathscr{G}_{n} \subset \mathscr{G}_{n+1}$. We will be concerned with the pull-back of $\mathscr{G}_{n}$ to the generic points of morphisms $f: T \rightarrow S$, where $T$ is a trait. ${ }^{3}$ In other words, if $\mu$ is the generic point of $T$ and $f(\mu) \in U$, then we can define the pull-back $\mathscr{G}_{n, \mu}$ of $\mathscr{G}_{n}$ to $\mu$. This extends to a quasi-finite flat subgroup scheme of the base changed semi-abelian scheme $\mathscr{C}_{T}$. Let it be $\mathscr{G}_{n, T}$. Therefore we can take the finite part

$$
\widetilde{\mathscr{I}}_{n, T}=\widetilde{\mathscr{C}\left[p^{n}\right]_{T}} \cap \mathscr{G}_{n, T} .
$$

We are interested in the behavior of the family $\left\{\widetilde{\mathscr{G}}_{n, T}\right\}_{n}$.

Proposition 2.7. There exist a finite sequence of blow-ups $\widetilde{S} \rightarrow S$ (centered over the closed point), a big open subset $V$ of $\widetilde{S}$ and a positive integer $n_{1} \geq 1$ such that, for every morphism $f: T \rightarrow S$, where $T$ is a trait, which factors through $V$ and sends the generic point $\mu \in T$ to $U$, the quotients $\widetilde{\mathscr{G}}_{n+n_{1}, T} / \widetilde{\mathscr{G}}_{n_{1}, T}$ define a Barsotti-Tate subgroup of $\mathscr{b}_{T} / \widetilde{\mathscr{G}}_{n_{1}, T}$. The conclusion remains true if one replaces $n_{1}$ by a larger integer.

Proof. We first define the finite part of $\mathscr{G}_{n}$ over $U$. It is the finite flat closed subgroup of $\widetilde{\mathscr{C}\left[p^{n}\right]_{\mid U}}$ defined by

$$
\widetilde{\mathscr{G}}_{n}=\mathscr{G}_{n} \cap \widetilde{\mathscr{C}\left[p^{n}\right]_{\mid U}} \text {. }
$$

This family may not constitute a Barsotti-Tate group. However there is a

$\left({ }^{3}\right)$ We define a trait (in a non-standard manner) as a 1-dimensional, local, henselian, regular and noetherian scheme. 
sequence of closed immersions

$$
\frac{\widetilde{\mathscr{G}}_{n+1, \eta}}{\widetilde{\mathscr{G}}_{n, \eta}} \stackrel{p}{\hookrightarrow} \frac{\widetilde{\mathscr{G}}_{n, \eta}}{\widetilde{\mathscr{G}}_{n-1, \eta}} \stackrel{p}{\hookrightarrow} \ldots \stackrel{p}{\hookrightarrow} \widetilde{\mathscr{G}}_{1, \eta} .
$$

Because $\widetilde{\mathscr{G}}_{1, \eta}$ is a noetherian scheme, this sequence stabilizes at some point. Therefore, for some integer $n_{0}$, the groups $\left\{Q_{n}:=\widetilde{\mathscr{G}}_{n+n_{0}, \eta} / \widetilde{\mathscr{G}}_{n_{0}, \eta}\right\}_{n}$ form a Barsotti-Tate group. Observe these groups extend to closed subgroups of the quotient semi-abelian scheme $\mathscr{C}_{\mid U} / \widetilde{\mathscr{G}}_{n_{0}}$, but they may not extend over S. By a variant of Gabber's lemma [18, Thm 2.1, Rmk. 2.2], there exists a sequence of blow-ups $\widetilde{S} \rightarrow S$ centered over the closed point $y$ such that $\mathscr{C}_{\mid U} / \widetilde{\mathscr{G}}_{n_{0}}$ extends to a semi-abelian scheme $\mathscr{B} \rightarrow \widetilde{S}^{4}$ We can and (for later purposes) we will assume that we have effected at least one blow-up. Remark that the base $\widetilde{S}$ is still regular, integral and noetherian of dimension 2 and generic characteristic 0. By a theorem of Raynaud [27, p.130], [14, Chap. I, Prop. 2.7], the natural commutative diagram of isogenies

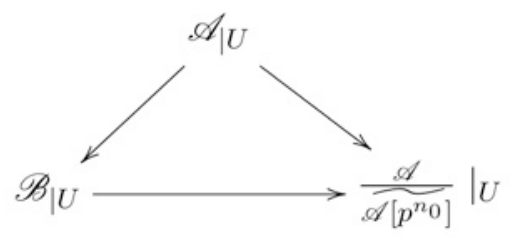

extends over $\widetilde{S}^{5}$ The kernel of the extended isogeny $\mathscr{C}_{\tilde{S}} \rightarrow \mathscr{B}$ is a quasifinite flat closed subgroup of $\left.\widetilde{\mathscr{C}\left[p^{n_{0}}\right.}\right]_{\tilde{S}}$, hence finite. It is an extension of $\widetilde{\mathscr{G}}_{n_{0}}$ over $\widetilde{S}$ and we use the same notation for it. Thus, we can write $\mathscr{B}=\mathscr{A}_{\tilde{S}} / \widetilde{\mathscr{G}}_{n_{0}}$. Observe now that

$$
Q_{n}=\frac{\widetilde{\mathscr{G}}_{n+n_{0}, \eta}}{\widetilde{\mathscr{G}}_{n_{0}, \eta}} \subseteq \frac{\left.\widehat{\mathscr{C [ p ^ { n + n _ { 0 } }}}\right]_{\tilde{S}}}{\widetilde{\mathscr{G}}_{n_{0}}},
$$

and the last quotient is a finite flat subgroup of $\mathscr{B}$. Hence Proposition 2.1 applies to the semi-abelian scheme $\mathscr{B}$ and the Barsotti-Tate subgroup $\left\{Q_{n}\right\}_{n}$ of $\mathscr{B}\left[p^{\infty}\right]_{\eta}$. Namely, there exists a big open subset $V$ of $\widetilde{S}$ and an integer $m \geq 0$ such that $Q_{m}$ extends to a finite flat subgroup $Q_{m}$ of $\mathscr{B}_{\mid V}$, the quotients $Q_{n+m} / Q_{m}$ extend to finite flat subgroups $\mathscr{H}_{n}$ of $\mathscr{B}_{\mid V} / \mathscr{Q}_{m}$ and the sequence $\left\{\mathscr{H}_{n}\right\}_{n}$ defines a Barsotti-Tate group.

$\left({ }^{4}\right)$ In this version of Gabber's lemma, it is only required that $S$ be quasicompact, excellent, reduced and normal. This is satsified under our assumptions.

${ }^{5}$ ) For Raynaud's theorem we only need normality of $\widetilde{S}$. 
Let now $f: T \rightarrow S$ be as in the statement, with $V$ given by the preceding construction. Because the generic point $\mu$ is sent into $U$, we can pull-back $\widetilde{\mathscr{I}}_{n}$ over $U$ to the generic point. By the functoriality of the finite part construction with respect to morphisms of local henselian schemes, this coincides with the previously constructed $\widetilde{\mathscr{G}}_{n, \mu}$. Furthermore, because $f$ factors through $V$, the preceding discussion for the quotients $Q_{n+m} / Q_{m}$ shows that, putting $n_{1}=n_{0}+m$, the family $\left\{\widetilde{\mathscr{G}}_{n+n_{1}, T} / \widetilde{\mathscr{G}}_{n_{1}, T}\right\}$ defines a Barsotti-Tate subgroup of $\mathscr{C}_{T} / \widetilde{\mathscr{G}}_{n_{1}, T} \cdot{ }^{6} \mathrm{We}$ stress the important fact that the index $n_{1}$ does not depend on the morphism $f$.

For the last claim of the proposition, we refer to Remark 2.2.

REMARK 2.8. It follows from the proof that we may suppose that the sequence of blow-ups $\widetilde{S} \rightarrow S$ is non-trivial: namely, it consists of at least one blow-up.

Construction 2.9. Let us place ourselves under the assumptions of Proposition 2.7. We consider a particular class of morphisms $f: T \rightarrow \widetilde{S}$, obtained as follows. Recall we assumed that $\widetilde{S}$ is obtained after at least one blow-up. Therefore we have a decomposition

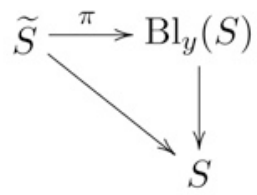

where $\mathrm{Bl}_{y}(S)$ is the blow-up of $S$ along $y$. The image in $\mathrm{Bl}_{y}(S)$ of the exceptional divisor of $\pi$ is a finite closed subscheme $F$ over $y$. The big open subset $\mathrm{Bl}_{y}(S) \backslash F$ of $\mathrm{Bl}_{y}(S)$ naturally embeds into $\widetilde{S}$. We may form

$$
W=V \cap\left(\mathrm{Bl}_{y}(S) \backslash F\right) .
$$

This is a big open subset of $\mathrm{Bl}_{y}(S)$. A morphism $f: T \rightarrow S$ that factors through $W \subset \mathrm{Bl}_{y}(S)$ also factors through $V \subset \widetilde{S}$.

2.3. We now apply the considerations in 2.2 to semi-abelian schemes over arithmetic surfaces.

$\left.{ }^{6}\right)$ Compare with the discussion in [15, pp. 134-136]. 


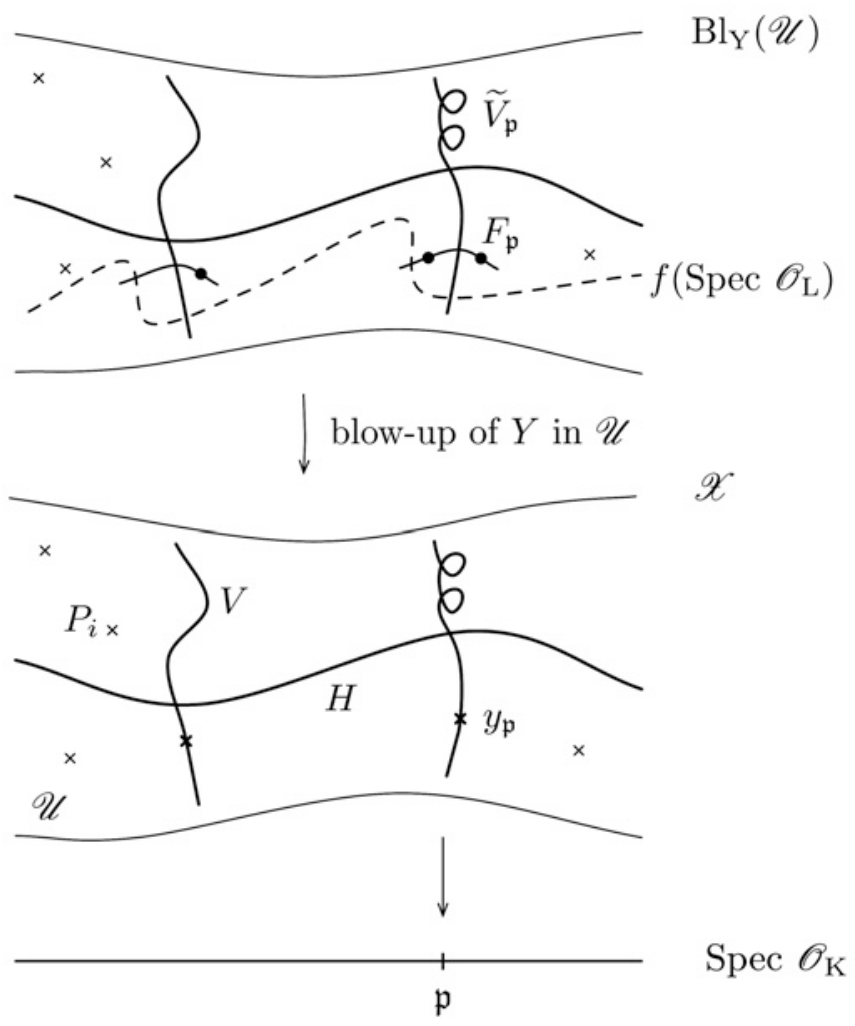

Fig. 1. The data in Theorem 2.10.

Let $K$ be a number field, $\mathcal{O}_{K}$ its ring of integers, $S=\operatorname{Spec} \mathcal{O}_{K}$. Let $\mathscr{\mathscr { C }}$ be a proper arithmetic surface over $S$, namely a regular, flat and proper scheme over $S$, with geometrically irreducible generic fiber of dimension 1 . Suppose given a semi-abelian scheme $\mathscr{b} \rightarrow \mathscr{C}$, where $\mathscr{C} b$ is a big open subset of $\mathscr{C}$ with generic point $\eta$, namely the complement of a finite set $\left\{P_{i}\right\}_{i \in I}$ of closed points in $\mathscr{X}$. We assume that $\mathscr{C}_{\eta}$ is abelian. Let $D \subset \mathscr{C}$ be the divisor of bad reduction of $\mathscr{C}$. We decompose $D=H+V$ into horizontal and vertical components. Define $\mathscr{V}=\mathscr{\mathscr { C }} \backslash D$. Let $\left\{\mathscr{G}_{n}\right\}_{n}$ be a Barsotti-Tate subgroup of $\mathscr{A}\left[p^{\infty}\right]_{\mathscr{C}}$. For every non-zero prime $\mathfrak{p}$ of $\mathcal{O}_{K}$ such that $V_{\mathfrak{p}}=V \cap \mathscr{C}_{\mathfrak{p}} \neq \emptyset$, we choose a closed point $y_{\mathfrak{p}} \in V_{\mathfrak{p}} \backslash H$. This provides a finite collection of closed points $Y=\left\{y_{\mathfrak{p}}\right\}_{\mathfrak{p} \in \mathscr{P}}$. Introduce $S_{\mathfrak{p}}=\operatorname{Spec} \widehat{\mathcal{O}_{\mathscr{C}, y_{\mathfrak{p}}}}$. We still denote $y_{\mathfrak{p}}$ its closed point. The Barsotti-Tate group $\left\{\mathscr{G}_{n}\right\}_{n}$ can be base-changed into a Barsotti-Tate group over the generic point of $S_{\mathfrak{p}}$. Let us denote by $W_{\mathfrak{p}}$ the big open subscheme of 
$\mathrm{Bl}_{y_{\mathfrak{p}}}\left(S_{\mathfrak{p}}\right)$ provided by Construction 2.9 applied to these data (see equation (2.1)). We have morphisms

$$
\phi_{\mathfrak{p}}: \mathrm{Bl}_{y_{\mathfrak{p}}}\left(S_{\mathfrak{p}}\right) \longrightarrow \mathrm{Bl}_{Y}(\mathscr{2 6})
$$

which lift the natural morphisms $S_{\mathfrak{p}} \rightarrow \mathscr{C}$. Let $F_{\mathfrak{p}}=\phi_{\mathfrak{p}}\left(\mathrm{Bl}_{y_{\mathfrak{p}}}\left(S_{\mathfrak{p}}\right) \backslash W_{\mathfrak{p}}\right)$, which is a finite closed subset of $\mathrm{Bl}_{Y}(\mathscr{Q C})$. By construction, $Y \cap H=\emptyset$ and $H$ can be seen as a divisor in $\mathrm{Bl}_{Y}(\mathscr{C})$. Write $\widetilde{V}_{\mathfrak{p}}$ for the strict transform of $V_{\mathfrak{p}}$. We then define the open subset of $\mathrm{Bl}_{Y}(\mathscr{C})$

$$
\mathscr{\mathscr { V }}=\mathrm{Bl}_{Y}(\mathscr{Q} 6) \backslash\left(H \cup \bigcup_{\mathfrak{p} \in \mathscr{P}}\left(F_{\mathfrak{p}} \cup \widetilde{V}_{\mathfrak{p}}\right)\right) .
$$

There is a natural inclusion of open subsets $\mathscr{T} \subset \mathscr{\mathscr { V }}$. An important feature of this construction is that the structure map $\mathrm{Bl}_{Y}(\mathscr{X}) \rightarrow S$ is surjective when restricted to $\mathscr{\mathscr { V }}$.

Let now $f:$ Spec $\mathcal{O}_{L} \rightarrow \mathscr{W}$ be an integral point of $\mathscr{\mathscr { W }}$ (in other words, $L$ is a finite extension of $K, \mathcal{O}_{L}$ its ring of integers, and $f$ a morphism of schemes over Spec $\left.\mathcal{O}_{K}\right)^{7}$. In particular it provides an integral point of $\mathscr{C} \backslash H$. For every prime $\mathfrak{q}$ of $\mathcal{O}_{L}$ lying above $\mathfrak{p} \in \mathscr{P}, f$ induces a morphism $f_{\mathfrak{q}}$ : Spec $\widehat{\mathcal{O}_{L, \mathfrak{q}}} \rightarrow S_{\mathfrak{p}}$ which factors through $W_{\mathfrak{p}}$. We also obtain by base change a semi-abelian scheme $\mathscr{b}_{q}$ over $\widehat{\mathcal{O}_{L, q}}$ and extended quasi-finite flat subgroups $\mathscr{G}_{n, \mathfrak{q}}$. Take the finite parts $\widetilde{\mathscr{G}}_{n, \mathrm{q}}$. By Proposition 2.7, there exists an integer $n_{1}$, which depends on $Y$ but not on $f$, such that the quotients $\left\{\widetilde{\mathscr{G}}_{n+n_{1}, \mathfrak{q}} / \widetilde{\mathscr{G}}_{n_{1}, \mathfrak{q}}\right\}_{n}$ define a Barsotti-Tate group. For any other prime $\mathfrak{q}$ not lying above $\mathscr{P}$, the analogous conclusion, with the same index $n_{1}$, is trivially true. Indeed $\mathfrak{q}$ is sent by $f$ into $\mathscr{T}$ and $\left\{\mathscr{G}_{n}\right\}_{n}$ is assumed to be a BarsottiTate subgroup of the abelian scheme $\mathscr{C} \mid \mathscr{r}$.

We summarize the preceding observations into the following theorem.

THeOREM 2.10. Let $\mathscr{X} \rightarrow$ Spec $\mathcal{O}_{K}$ be an arithmetic surface and $\mathscr{C}$ a semi-abelian scheme over a big open subset $\mathscr{Z b}$ of $\mathscr{K}$, generically abelian. Let $D$ be the divisor of bad reduction in $\mathscr{C b}$ and decompose $D=H+V$ in horizontal and vertical components. Put $\mathscr{V}=\mathscr{b} \backslash D$, and choose a closed point $y_{\mathfrak{p}} \in V \cap \mathscr{C}_{\mathfrak{p}} \backslash H$ for every $\mathfrak{p}$ in $\mathscr{P}$, the subset of Spec $\mathcal{O}_{K} \backslash\{0\}$ defined by the $\mathfrak{p}$ such that $V \cap \mathscr{X}_{\mathfrak{p}} \neq \emptyset$.

Then, given a Barsotti-Tate subgroup $\left\{\mathscr{G}_{n}\right\}_{n}$ of $\mathscr{A}_{\mid \mathscr{V}}$, there exists an integer $n_{1} \geq 0$ such that for every integral point $f: \operatorname{Spec} \mathcal{O}_{L} \rightarrow \mathscr{Q b}$ factor-

$\left({ }^{7}\right)$ Because the structure morphism $\mathscr{W} \rightarrow S$ is surjective, Rumely's theorem ([29], [23]) will ensure that there exist infinitely many such points (for varying finite extensions $L$ of $K$ ). 
ing through $\mathscr{W}$ defined by (2.2), we have:

i. the image of $f$ is disjoint with the horizontal component $H$; in particular, the pull-back $f^{*}$ of is a semi-abelian scheme over $\mathcal{O}_{L}$, generically abelian.

ii. For every prime a of $\mathcal{O}_{L}$ lying above one of the primes $\mathfrak{p}$ in $\mathscr{P}$, denote by $\mathscr{A}_{\mathfrak{q}}$ the base change of $\mathscr{A}_{\mathrm{b}}$ to Spec $\widehat{\mathcal{O}_{L, q}}$ induced by $f$. Let $\mathscr{G}_{n, \mathfrak{q}}$ be the quasi-finite flat subgroup of $\mathscr{A}_{\mathfrak{a}}$ extending the base change of $\mathscr{G}_{n}$ to the generic point of Spec $\widehat{\mathcal{O}_{L, \mathfrak{q}}}$, and $\widetilde{\mathscr{G}}_{n, \mathfrak{q}}$ its finite part. Then the family $\left\{\widetilde{\mathscr{G}}_{n+n_{1}, \mathrm{q}} / \widetilde{\mathscr{G}}_{n_{1}}\right\}_{n}$ defines a Barsotti-Tate subgroup of $\mathscr{b}_{\mathfrak{q}} / \widetilde{\mathscr{I}}_{n_{1}}$.

\section{Some diophantine inequalities for heights}

In this section we discuss some generalities and diophantine inequalities for heights of cycles in arithmetic varieties, with respect to hermitian line bundles whose metrics are of some logarithmic singular type. This kind of heights will appear in later considerations, when dealing with minimal compactifications of moduli spaces of abelian varieties. The arithmetic intersection formalism leading to these heights has been developed by Burgos-Kramer-Kühn [6]. Their finiteness property has been explored by the second author of the present article in [16]. A diophantine inequality, generalizing results by Zhang [36] in the spirit of Faltings' lemma [12, Lemma 3] on heights defined by means of metrics with logarithmic singularities, has been established in some unpublished work by the first author [4]. Here we provide the precise statements and some details.

3.1. We introduce the heights and formulate the finiteness property we will need. Let $\mathscr{C}$ and $\mathscr{Y}$ be arithmetic varieties over the ring of integers $\mathcal{O}_{K}$ of a number field $K$, namely, projective and flat schemes over Spec $\mathcal{O}_{K}$. We make the following geometric assumptions:

- the generic fiber $\mathscr{Y}_{K}$ is smooth over $K$;

- there is a surjective morphism $\varphi: \mathscr{Y} \rightarrow \mathscr{C}$ the restriction of which to the generic fiber induces an isomorphism $\mathscr{Y}_{K} \backslash \varphi_{K}^{-1}(F) \stackrel{\sim}{\rightarrow} \mathscr{C}_{K} \backslash F$, where $F$ is a Zariski closed subset of $\mathscr{C}_{K}$ and $D=\varphi_{K}^{-1}(F)_{\text {red }}$ is a divisor such that $D(\mathbb{C})$ has normal crossings. 
In particular, $U=\mathscr{C}_{K} \backslash F$ is contained in the regular locus $\mathscr{X}_{K}^{\text {reg }}$ and

$$
U(\mathbb{C}):=\coprod_{\sigma: K \hookrightarrow \mathrm{C}} U_{\sigma}(\mathbb{C})
$$

is a complex quasi-projective manifold. Let $\mathscr{B}$ be a line bundle on $\mathscr{X}$ and $\|\cdot\|$ a smooth hermitian metric on $\mathscr{L}_{\mid U(\mathrm{C})}$, invariant under the action of complex conjugation. We denote by $\overline{\mathscr{C}}=(\mathscr{L},\|\cdot\|)$ this singular hermitian line bundle in the sense of Arakelov geometry.

Definition 3.1. We say that $\overline{\mathscr{B}}$ is a pre-log-log hermitian line bundle, with singularities along $F$, with respect to $\varphi$, if the pull-back singular hermitian line bundle $\varphi^{*} \mathscr{\mathscr { C }}$ is a pre-log-log hermitian line bundle on $\mathscr{F}$, with singularities along $D$ [6, Sec. 7], [16, Sec. 3]. Similarly, one defines the notion of continuous or smooth hermitian line bundle on $\mathscr{X}$.

Under the pre-log-log assumption, according to [6, Sec. 7], there is a well defined height function $h_{\varphi^{*} \overline{\mathscr{C}}}$ on algebraic cycles on $\mathscr{Y}$ whose flat irreducible components intersect $D$ properly (or equivalently, are not contained in $D$ ) over $\mathscr{Y}_{K}$. We refer to loc. cit. for details, as well as to [16, Sec. 6 . We observe that the constructions of height functions in these references extend to non-necessarily regular arithmetic varieties, with smooth generic fibers. We refer to [5, Rmk ii, p. 941] in the case of smooth hermitian metrics and to [7, Sec. 4.3] for the general case.

The theory of heights with respect to semi-positive continuous hermitian line bundles is developed in [21, Sec. 5]. We will only need the case of an ample line bundle $\mathscr{C}$ on $\mathscr{C}$ endowed with a continuous metric $\|\cdot\|_{1}$ with semi-positive first Chern current (after pull-back to $\mathscr{H}$ ). Then, $\varphi^{*} \overline{\mathscr{B}}_{1}$ is an integrable hermitian line bundle on $\mathscr{Y}$, in the sense of [21, Def. 4.7.1], and the height function $h_{\varphi^{*} \overline{\mathscr{C}}_{1}}$ is defined. The integrability property requires an easy proof, that we give in Lemma 3.7 below.

Let us denote by $Z_{p, U}(\mathscr{C})$ the free abelian group of $p$-dimensional cycles on $\mathscr{X}$, whose flat irreducible components intersect the open subset $U=\mathscr{C}_{K} \backslash F$ of $\mathscr{C}_{K}$. We similarly define $Z_{p, \varphi^{-1}(U)}(\mathscr{H})$. To a pre-log-log (resp. smooth, ample continuous semi-positive) hermitian line bundle $\overline{\mathscr{B}}$, we attach a height function

$$
h_{\overline{\mathscr{C}}}: \bigoplus_{p} Z_{p, U}(\mathscr{C}) \longrightarrow \mathbb{R}
$$

characterized by the following properties:

- $h_{\bar{L}}$ is Z-linear; 
- $h_{\varphi^{*} \overline{\mathscr{C}}}(z)=h_{\overline{\mathscr{C}}}\left(\varphi_{*} z\right)$, where $\varphi_{*}$ is the usual push-forward morphism of cycles

$$
\varphi_{*}: \bigoplus_{p} Z_{p, \varphi^{-1}(U)}(\mathscr{Y}) \longrightarrow \bigoplus_{p} Z_{p, U}(\mathscr{B}) .
$$

If $z \in Z_{p, U}(\mathscr{X})$ is a cycle such that $\operatorname{deg}_{\mathscr{C}_{K}} z_{K} \neq 0$, its normalized height is defined by

$$
\widetilde{h}_{\overline{\mathscr{L}}}(z)=\frac{h_{\overline{\mathscr{C}}}(z)}{[K: \mathrm{Q}] \operatorname{deg}_{\mathscr{C}_{K}} z_{K}} .
$$

Some properties of these height functions, such as compatibility with change of ground ring, are straightforward consequences of the corresponding properties for $h_{\varphi^{*} \overline{\mathscr{b}}}$.

The next statement is adapted from [16, Thm. 1.3], and may be seen as a Liouville type inequality for the approximation of $F$ by cycles on $\mathscr{Y}$.

Theorem 3.2. Let $\mathscr{X}, \mathscr{Y}, \varphi: \mathscr{Y} \rightarrow \mathscr{B}$ be as above, and $\overline{\mathscr{C}}$ a pre-log-log hermitian line bundle on $\mathscr{C}$ with singularities along $F$, with respect to $\varphi$. Assume that $\mathscr{B}$ is ample over $\mathscr{B}$ and that the first Chern form $\mathrm{c}_{1}(\overline{\mathscr{B}})$ is semi-positive on $U(\mathrm{C})$. Then, for any smooth hermitian metric $\|\cdot\|_{0}$ on $\mathscr{B}$ with semi-positive first Chern form (after pull-back to $\mathscr{Y}$ ), there exist real constants $\alpha, \beta, \gamma>0$ such that, for every effective cycle $z \in Z_{p, U}(\mathscr{B})$, such that $\operatorname{deg}_{\mathscr{L}_{K}} z_{K} \neq 0$ we have:

$$
\widetilde{h}_{\overline{\mathscr{B}}_{0}}(z)+\gamma>0
$$

and

$$
\left|\widetilde{h}_{\overline{\mathscr{C}}}(z)-\widetilde{h}_{\overline{\mathscr{L}}_{0}}(z)\right| \leq \alpha+\beta \log \left(\widetilde{h}_{\overline{\mathscr{L}}_{0}}(z)+\gamma\right) .
$$

REMARK 3.3. Since the line bundle $\mathscr{B}$ is ample, there exist smooth metrics on $\mathscr{L}$ with semi-positive first Chern form. For example, one may consider a very ample power $\mathscr{L}^{N}$ and use the restriction of the FubiniStudy metric on the sheaf $\mathcal{O}(1)$ of the projective space $\mathrm{P}\left(H^{0}\left(\mathscr{X}, \mathscr{L}^{N}\right)\right)$. For this particular metric $\|\cdot\|_{0}$, a multiple of the height $h_{\overline{\mathscr{L}}_{0}}$ is the restriction to cycles on $\mathscr{C}$ of the usual Faltings height on $\mathrm{P}\left(H^{0}\left(\mathscr{C}, \mathscr{L}^{N}\right)\right)$. Any other height for a pre-log-log metric (and in particular for a smooth metric) on $\mathscr{b}$ then differs from $h_{\overline{\mathscr{L}}_{0}}$ by an archimedean factor, expressed by integrals of so-called Bott-Chern secondary forms.

Proof of Theorem 3.2. The proof follows the lines of [16, Sec. 6.2], with several simplifications. The idea is to adapt the argument due to 
Faltings, for heights of points, to the higher dimensional situation [12, Lemma 3], to reduce to an application of Jensen's inequality. We sketch the proof and refer to loc. cit. for more details. First of all, by assumption $\mathscr{B}$ is ample. After possibly replacing it by some positive power, we find global sections $s_{1}, \ldots, s_{m} \in H^{0}(\mathscr{X}, \mathscr{L})$ such that

$$
F(\mathrm{C})=\left(\operatorname{div} s_{1}\right)(\mathrm{C}) \cap \ldots \cap\left(\operatorname{div} s_{m}\right)(\mathrm{C}) .
$$

One may suppose that the cycle $z$ is flat, integral and properly intersects div $s_{1}$ over C. By the definition of the height, one is reduced to bound integrals of the form

$$
\int_{\varphi^{-1}(z(\mathrm{C}))}\left|\varphi^{*}(f)\right| \mathrm{c}_{1}\left(\varphi^{*} \overline{\mathscr{L}}\right)^{i} \mathrm{c}_{1}\left(\varphi^{*} \overline{\mathscr{L}}_{0}\right)^{j}, \quad i+j=\operatorname{dim} z_{K} .
$$

Here we wrote $\|\cdot\|=e^{-f / 2}\|\cdot\|_{0}$, and we note that the integral has to be computed on a suitable resolution of singularities of $\varphi^{-1}(z(\mathbb{C})$ ). Recall that $z_{K}$ meets properly div $s_{1}$, that the metric $\|\cdot\|$ has logarithmic singularities, and that the first Chern forms are semi-positive. After a rescaling of the smooth metric, we may thus replace $\left|\varphi^{*}(f)\right|$ by $\log \log \left\|\varphi^{*} s_{1}\right\|_{0}^{-2}$. This rescaling can be made independently of $z$. (Observe that, by comparison to loc. cit., there is an important simplification in this step. Namely the positivity assumption made on the first Chern forms allows to avoid the decomposition result in Theorem 4.3. ${ }^{8}$ ) Now, one proceeds to decrease the exponent $i$. For this, we first write

$$
\mathrm{c}_{1}\left(\varphi^{*} \overline{\mathscr{C}}\right)=\mathrm{c}_{1}\left(\varphi^{*} \overline{\mathscr{L}}_{0}\right)+d d^{c} f
$$

on $\varphi^{-1}(U(\mathrm{C}))$. One is reduced to bound integrals of the form

$$
\int_{\varphi^{-1}(z(\mathrm{C}))} \log \log \left\|\varphi^{*} s_{1}\right\|_{0}^{-2} \cdot d d^{c} f \cdot \mathrm{c}_{1}\left(\varphi^{*} \overline{\mathscr{L}}\right)^{i-1} \cdot \mathrm{c}_{1}\left(\varphi^{*} \overline{\mathscr{L}}_{0}\right)^{j} .
$$

In this situation, one can apply Stokes theorem. Taking into account the following relation of differential forms outside $F(\mathrm{C})$

$$
-d d^{c} \log \log \left\|s_{1}\right\|_{0}^{-2}=\frac{i}{2 \pi} \frac{\partial \log \left\|s_{1}\right\|_{0}^{-2} \wedge \bar{\partial} \log \left\|s_{1}\right\|_{0}^{-2}}{\left(\log \left\|s_{1}\right\|_{0}^{-2}\right)^{2}}-\frac{c_{1}\left(\overline{\mathscr{L}}_{0}\right)}{\log \left\|s_{1}\right\|_{0}^{-2}},
$$

$\left.{ }^{8}\right)$ With the notations of [16, Thm. 1.3], this explains that we can take $r=1$. 
the positivity of the Chern differential forms, and elementary bounds between functions with logarithmic singularities, one reduces to bound integrals of the form

$$
-\frac{i}{2 \pi} \int_{\varphi^{-1}(z(\mathrm{C}))} \partial\left(\frac{1}{\left(\log \left\|\varphi^{*} s_{1}\right\|_{0}^{-2}\right)^{1 / 2}}\right) \wedge \bar{\partial} \log \left\|\varphi^{*} s_{1}\right\|_{0}^{-2} c_{1}\left(\varphi^{*} \overline{\mathscr{C}}\right)^{i-1} c_{1}\left(\varphi^{*} \overline{\mathscr{L}}_{0}\right)^{j} .
$$

Such integrals are treated in loc. cit., Lemma 5.5. After reducing the exponent $i$ to 0 , the main term to bound is an integral of the form

$$
\int_{\varphi^{-1}(z(\mathrm{C}))} \log \log \left\|\varphi^{*} s_{1}\right\|_{0}^{-2} \mathrm{c}_{1}\left(\varphi^{*} \overline{\mathscr{L}}_{0}\right)^{d} .
$$

By Jensen's inequality, this is bounded in terms of

$$
\log \left(\int_{\varphi^{-1}(z(\mathrm{C}))} \log \left\|\varphi^{*} s_{1}\right\|_{0}^{-1} \frac{\mathrm{c}_{1}\left(\varphi^{*} \overline{\mathscr{L}}_{0}\right)^{d}}{[K: \mathrm{Q}] \operatorname{deg}_{\mathscr{L}_{K}} z_{K}}\right) .
$$

Finally, by the definition of the height and the existence of a universal lower bound for normalized heights with respect to ample smooth hermitian line bundles, this last term is bounded by $\log \left(\widetilde{h}_{\overline{\mathscr{C}}_{0}}(z)+\gamma\right)$, where the constant $\gamma$ only depends on the smooth metric $\|\cdot\|_{0}$. This completes the proof.

COROLLARY 3.4. Let $\overline{\mathscr{C}}$ be an ample pre-log-log hermitian line bundle on $\mathscr{C}$ with semi-positive first Chern form. Then, given a real number $A>0$, there exist finitely many effective cycles $z \in Z_{p, U}(\mathscr{C})$ with $h_{\overline{\mathscr{C}}}(z) \leq A$ and $\operatorname{deg}_{\mathscr{L}_{K}} z_{K} \leq A$.

Proof. After possibly replacing $\mathscr{B}$ by a high power, we may assume that $\mathscr{L}$ is very ample. Then, using a projective embedding and the FubiniStudy metric on projective space, we can construct a smooth hermitian metric on $\mathscr{L}$, say $\|\cdot\|_{0}$, with positive first Chern form and, more importantly, such that the height $h_{\overline{\mathscr{L}}_{0}}$ is the restriction of some usual Faltings height for the ambient projective space. Then the finiteness property is an immediate consequence of the comparison theorem above and the finiteness property for $h_{\overline{\mathscr{L}}_{0}}$ proven by Bost-Gillet-Soulé in [5, Thm. 3.2.5].

3.2. We now proceed to some generalization of Zhang's inequality [36, Thm. 5.2], [1, Thm. 2.4] for the height functions with respect to prelog-log hermitian line bundles. This will be applied to prove bounds for 
cycles in moduli spaces of abelian varieties, in terms of heights of conveniently chosen integral points.

Theorem 3.5. Let $\mathscr{X}, \mathscr{Y}, \varphi: \mathscr{Y} \rightarrow \mathscr{C}$ be as above. Let $\overline{\mathscr{C}}$ be a pre-loglog hermitian line bundle on $\mathscr{C}$ with semi-positive first Chern form. Assume that $\mathscr{B}$ is ample and the unbounded locus of the metric is exactly $F(\mathrm{C}) .{ }^{9}$ Then there is an inequality

$$
\widetilde{h}_{\overline{\mathscr{L}}}(\mathscr{C}) \leq d \sup _{V \subseteq U} \inf _{x \in V(\bar{K})} \widetilde{h}_{\overline{\mathscr{L}}}(x),
$$

where $d$ is the Krull dimension of $\mathscr{C}$ and the supremum runs over all open Zariski subsets $V$ of $U=\mathscr{C}_{K} \backslash F$. Moreover the right hand side of the inequality is a finite quantity.

REMARK 3.6. In the statement of the theorem, the normalized height of an algebraic point $x \in V(\bar{K})$ has to be understood as follows. The point $x$ induces a 1 -dimensional horizontal cycle $z$ in $\mathscr{X}$. Then we put

$$
\begin{aligned}
\tilde{h}_{\mathscr{L}^{\prime}}(x): & =\widetilde{h}_{\overline{\mathscr{L}}}(z) \\
& =\frac{h_{\mathscr{S}^{\prime}}(z)}{[K: \mathrm{Q}] \operatorname{deg}_{\mathscr{C}_{K}} z_{K}}=\frac{h_{\overline{\mathscr{S}}}(z)}{[K(x): \mathrm{Q}]} .
\end{aligned}
$$

To prove Theorem 3.5, we will truncate the pre-log-log hermitian metric and work with continuous hermitian metrics with semi-positive first Chern current. We will need two lemmas.

Lemma 3.7. Let $\|\cdot\|_{1}$ (resp. $\|\cdot\|$ ) be a continuous (resp. pre-log-log) hermitian metric on the ample line bundle $\mathscr{L}$, whose first Chern current is semi-positive. Assume that $\|\cdot\|_{1} \leq\|\cdot\|$ on $U(\mathbb{C})$. Then:

i. $\varphi^{*} \overline{\mathscr{L}}_{1}$ is an integrable hermitian line bundle;

ii. for every effective cycle $z \in Z_{p, U}(\mathscr{C})$ we have the inequality

$$
h_{\overline{\mathscr{S}}}(z) \leq h_{\overline{\mathscr{L}}_{1}}(z) \text {. }
$$

Proof. Let $\overline{\mathscr{b}}$ be a hermitian ample line bundle on $\mathscr{F}$, with a smooth hermitian metric whose first Chern form is strictly positive. For every integer $N \geq 1$, the continuous hermitian line bundle $\varphi^{*}\left(\overline{\mathscr{C}}_{1}\right)^{N} \otimes \overline{\mathscr{C}}$

$\left({ }^{9}\right)$ These hypotheses could be slightly weakened, but this formulation is enough for our purposes. 
is ample on the generic fiber and has strictly positive first Chern current. By [21, Thm. 4.6.1], $\varphi^{*}\left(\overline{\mathscr{D}}_{1}\right)^{N} \otimes \overline{\mathscr{C}}$ is admissible [21, Def. 4.5.5] and there exists an increasing sequence of smooth metrics $\|\cdot\|_{k}$ uniformly converging to the metric on $\varphi^{*}\left(\overline{\mathscr{C}}_{1}^{N}\right) \otimes \overline{\mathscr{b}}$, and with semi-positive first Chern forms. In particular, in the case $N=1$, it follows that $\varphi^{*} \overline{\mathscr{B}}_{1}=$ $\left(\varphi^{*} \overline{\mathscr{L}}_{1} \otimes \overline{\mathscr{C}}\right) \otimes \overline{\mathscr{b}}^{-1}$ is integrable, thus proving $i$. Also, as the sequence is increasing, these smooth metrics are smaller than the pre-log-log metric on $\varphi^{*}\left(\overline{\mathscr{C}}^{N}\right) \otimes \overline{\mathscr{b}}$, which has positive first Chern current. From this and the definition of height easily follows the inequality

$$
h_{\varphi^{*}\left(\overline{\mathscr{C}}^{N}\right) \otimes \overline{\mathscr{b}}}(z) \leq h_{\varphi^{*}\left(\mathscr{C}^{N}\right) \otimes \cdot \mathscr{C}_{k}}(z)
$$

for $z \in Z_{p, U}(\mathscr{C})$ effective. The right hand side of the inequality converges to $h_{\varphi^{*}\left(\overline{\mathscr{L}}_{1}\right)^{N} \otimes \overline{\mathscr{C}}}(z)$ by [21, Thm. 5.5.2]. Working on the definition of height, we thus obtain

$$
N^{d} h_{\varphi^{*} \overline{\mathscr{C}}}(z) \leq N^{d} h_{\varphi^{*} \overline{\mathscr{C}}_{1}}(z)+O\left(N^{d-1}\right),
$$

where the $O$ term only depends on several arithmetic intersection numbers on $z$. The assertion $i i$ now follows by dividing these heights by $N^{d}$ and letting $N$ converge to $\infty$.

Lemma 3.8. The statement of Theorem 3.5 holds for continuous hermitian metrics $\|\cdot\|_{1}$ on $\mathscr{L}$ with semi-positive first Chern current.

Proof. The original argument of [36] can be adapted to this case, because $\mathscr{L}$ is ample and the curvature current of $\overline{\mathscr{L}}_{1}$ is semi-positive. For this, one needs to refer to the version of the arithmetic Hilbert-Samuel theorem proven by Randriambololona [26]. Observe that this work already deals with the case when the generic fiber $\mathscr{C}_{K}$ is just reduced, non-necessarily smooth. By construction, the arithmetic intersection number in the dominant term of the main theorem in loc. cit., coincides with our height with respect to $\overline{\mathscr{B}}_{1}$.

We are now ready to prove Theorem 3.5.

Proof of Theorem 3.5. It suffices to show the inequality with $\mathscr{B}$ replaced by some positive power $\mathscr{L}^{N}$. Thus we can suppose that $\mathscr{b}$ is very ample and that there exists a global section $s \in H^{0}(\mathscr{C}, \mathscr{B})$ with $F(\mathbb{C}) \subseteq(\operatorname{div} s)(\mathrm{C})$.

Endow $\mathscr{L}$ with a smooth hermitian metric $\|\cdot\|_{0}$ with semi-positive first Chern form and such that $\|s\|_{0} \ll 1$. Write $\|\cdot\|=e^{-g / 2}\|\cdot\|_{0}$. Observe that the condition $d d^{c} \varphi^{*}(g)+c_{1}\left(\varphi^{*} \overline{\mathscr{C}}_{0}\right) \geq 0$ and the surjectivity of $\varphi$ guarantee 
that $g$ is locally bounded above. Since $\mathscr{C}(\mathrm{C})$ is compact, $g$ has a global upper bound. Because the inequality we aim to prove does not change after rescaling the metric, we can suppose that $g \leq 0$. Since $\|\cdot\|$ admits logarithmic singularities along $F(\mathbb{C})$, there exists a positive constant $A$ such that $-g \leq A \log \log \|s\|_{0}^{-1}$. For every integer $j \geq 3$, define

$$
g_{j}=\max \{g,-A \log \log j\} .
$$

Because we assume that the unbounded locus of $g$ is exactly $F(\mathbb{C})$, the functions $g_{j}$ are continuous on $\mathscr{C}(\mathrm{C})$. Because the maximum of two psh functions is already psh, we have $d d^{c} \varphi^{*}\left(g_{j}\right)+c_{1}\left(\varphi^{*} \mathscr{L}_{0}\right) \geq 0$. It is clear that the sequence $g_{j}$ decreases towards $g$. We define the continuous metrics on $\mathscr{B}$

$$
\|\cdot\|_{j}=e^{-g_{j} / 2}\|\cdot\|_{0} .
$$

They form an increasing sequence pointwise converging to $\|\cdot\|$ on $U(\mathrm{C})$, with semi-positive first Chern currents. By Lemma 3.7 we know that

$$
h_{\overline{\mathscr{B}}}(z) \leq h_{\overline{\mathscr{L}}_{j}}(z)
$$

for any effective cycle $z \in Z_{p, U}(\mathscr{C})$. Observe that by Lemma 3.8, this already guarantees the finiteness of the right hand side of inequality (3.1).

To establish (3.1), we reason by contradiction. Suppose the inequality (3.1) does not hold. Then, for some $\varepsilon>0$ we can find a generic sequence of points $\left\{x_{k}\right\}_{k \geq 1}$ in $(\mathscr{C} \backslash \operatorname{div}(s))(\bar{K})$ with

$$
d \widetilde{h}_{\overline{\mathscr{C}}}\left(x_{k}\right) \leq \widetilde{h}_{\overline{\mathscr{C}}}(\mathscr{B})-\varepsilon .
$$

By Theorem 3.2 and (3.3) we deduce that $\left\{\widetilde{h}_{\overline{\mathscr{L}}_{0}}\left(x_{k}\right)\right\}_{k}$ is bounded above by some constant $B$. On the other hand (3.2) applied to $\mathscr{C}$ and (3.3) give

$$
d \widetilde{h}_{\overline{\mathscr{L}}_{j}}\left(x_{k}\right) \leq d \widetilde{h}_{\overline{\mathscr{L}}_{j}}\left(x_{k}\right)-d \widetilde{h}_{\overline{\mathscr{S}}}\left(x_{k}\right)+\widetilde{h}_{\overline{\mathscr{L}}_{j}}(\mathscr{\mathscr { C }})-\varepsilon .
$$

Fix $k \geq 1$ and let $L$ be a field of definition of $x_{k}$. There are inequalities

$$
\begin{aligned}
\widetilde{h}_{\overline{\mathscr{S}}_{j}}\left(x_{k}\right)-\widetilde{h}_{\overline{\mathscr{S}}}\left(x_{k}\right) & =\frac{1}{[L: \mathrm{Q}]} \sum_{\sigma: L \hookrightarrow \mathrm{C}} \frac{g_{j}-g}{2}\left(x_{k, \sigma}\right) \\
& =\frac{1}{[L: \mathrm{Q}]} \sum_{\sigma: L \hookrightarrow \mathrm{C}} \frac{\max \{0,-g-A \log \log j\}}{2}\left(x_{k, \sigma}\right) \\
& \leq \frac{A}{2[L: \mathrm{Q}]} \sum_{\sigma: L \hookrightarrow \mathrm{C}} \max \left\{0, \log \log \|s\|_{0}^{-1}-\log \log j\right\}\left(x_{k, \sigma}\right) \\
& \leq \frac{A}{2} \log \left(1+\frac{1}{\log j} \frac{1}{[L: \mathrm{Q}]} \sum_{\sigma: L \hookrightarrow \mathrm{C}} \log \|s\|_{0}^{-1}\left(x_{k, \sigma}\right)\right) .
\end{aligned}
$$


To derive the last inequality, we made use of the Jensen's formula, again inspired by Faltings [12, Lemma 3]. By definition of height and because $\widetilde{h}_{\overline{\mathscr{S}}_{0}}\left(x_{k}\right) \leq B$, we finally obtain

$$
\begin{aligned}
\widetilde{h}_{\overline{\mathscr{L}}_{j}}\left(x_{k}\right)-\widetilde{h}_{\overline{\mathscr{L}}}\left(x_{k}\right) & \leq \frac{A}{2} \log \left(1+\frac{\widetilde{h}_{\overline{\mathscr{L}}_{0}}\left(x_{k}\right)}{\log j}\right) \\
& \leq \frac{A}{2} \log \left(1+\frac{B}{\log j}\right) .
\end{aligned}
$$

Fix $j \geq 3$ large enough so that

$$
\frac{A}{2} \log \left(1+\frac{B}{\log j}\right) \leq \frac{\varepsilon}{2 d} .
$$

Together with (3.4), we derive

$$
d \widetilde{h}_{\overline{\mathscr{L}}_{j}}\left(x_{k}\right) \leq \widetilde{h}_{\overline{\mathscr{C}}_{j}}(\mathscr{C})-\frac{\varepsilon}{2}
$$

for every point of the generic sequence $\left\{x_{k}\right\}_{k}$. But for the continuous metric $\|\cdot\|_{j}$, Zhang's inequality holds (Lemma 3.8). Hence (3.5) provides a contradiction, and the proof is complete.

3.3. As an illustration of the preceding constructions, in the final part of this section we present the geometric framework to which the theory of heights will be applied in the sequel.

Let $\ell$ be a positive integer with $\ell=\ell_{1} \ell_{2}$, where $\ell_{1}, \ell_{2} \geq 3$ are relatively prime. We first construct a suitable model over Spec $Z\left[\zeta_{\ell}\right]$ of the minimal compactification of the fine moduli space of principally polarized $g$-dimensional abelian varieties with level $\ell$ structure. We will appeal to the arithmetic minimal compactifications of Faltings-Chai [14, Chap. V, Thm.2.3, Thm. 2.5].

LEMMA 3.9. There exists a normal integral scheme $\boldsymbol{A}_{g, \ell}^{*}$, proper over Spec Z $\left[\zeta_{\ell}\right]$, and an ample line bundle $\omega$ on $\boldsymbol{A}_{g, \ell}^{*}$, characterized by the following properties:

$i$. the restrictions of $\boldsymbol{A}_{g, \ell}^{*}$ and $\omega$ to Spec $Z\left[\zeta_{\ell}, 1 / \ell\right]$ coincide with the minimal compactification and canonical ample line bundle of Faltings-Chai;

ii. the restriction of $\boldsymbol{A}_{g, \ell}^{*}$ to $\operatorname{Spec} Z_{[}\left[\zeta_{\ell}, 1 / \ell_{i}\right]$ admits a natural finite morphism to $\boldsymbol{A}_{g, \ell_{i}}^{*}(i=1,2)$, extending the canonical morphism $\boldsymbol{A}_{g, \ell} \rightarrow \boldsymbol{A}_{g, \ell_{i}}$;

iii. the restriction of $\omega$ to Spec $Z\left[\zeta_{\ell}, 1 / \ell_{i}\right]$ arises as a pull-back of the canonical line bundle of Faltings-Chai on $\boldsymbol{A}_{g, \ell_{i}}^{*}$, by the natural morphism, for $i=1,2$. 
Proof. We may assume $g \geq 2$, since the result easily follows from Deligne-Rapoport [9, Chap. V, Sec. 4] when $g=1$. Let us first construct $\boldsymbol{A}_{g, \ell}^{*}$, satisfying the requirements of the lemma. We recall that there is a natural finite and étale morphism of fine moduli schemes

$$
\boldsymbol{A}_{g, \ell} \longrightarrow \boldsymbol{A}_{g, \ell_{i}}[1 / \ell] \text {. }
$$

The notation $\boldsymbol{A}_{g, \ell_{i}}[1 / \ell]$ means we inverted the primes dividing $\ell$ in the structure sheaf of $\boldsymbol{A}_{g, \ell_{i}}$. In particular $\boldsymbol{A}_{g, \ell}$ is obtained by normalization of $\boldsymbol{A}_{g, \ell_{i}}[1 / \ell]$ in the function field of $\boldsymbol{A}_{g, \ell}$. We define $\widetilde{\boldsymbol{A}}_{g, \ell}^{(i)}$ as the normalization of $\boldsymbol{A}_{g, \ell_{i}}^{*}$ in the function field of $\boldsymbol{A}_{g, \ell}$. By construction, this is a normal integral scheme, proper of finite type over Spec $Z\left[\zeta_{\ell}, 1 / \ell_{i}\right]$. Moreover the natural morphism $\pi_{i}: \widetilde{\boldsymbol{A}}_{g, \ell}^{(i)} \rightarrow \boldsymbol{A}_{g, \ell_{i}}^{*}$ is finite because the base is an integral normal scheme and we are in generic characteristic 0 . We also define $\widetilde{\omega}_{i}=\pi_{i}^{*} \omega_{i}$, where $\omega_{i}$ is the canonical ample sheaf on $\boldsymbol{A}_{g, \ell_{i}}^{*}$ of Faltings-Chai [14, Thm. 2.5 (1)]. It will be enough to show that the restrictions of the $\widetilde{\boldsymbol{A}}_{g, \ell}^{(i)}\left(\right.$ resp. $\left.\widetilde{\omega}_{i}\right)$ to Spec $Z\left[\zeta_{\ell}, 1 / \ell\right]$ coincide with the arithmetic minimal compactification $\boldsymbol{A}_{g, \ell}^{*}$ (resp. the canonical sheaf $\omega$ on $\boldsymbol{A}_{g, \ell}^{*}$ ). This will also give the necessary gluing condition.

We claim that the arithmetic minimal compactification $\boldsymbol{A}_{g, \ell}^{*}$ (of FaltingsChai) is obtained from $\boldsymbol{A}_{g, \ell_{i}}^{*}[1 / \ell]$ by normalization too. This follows from the Koecher principle. Indeed, recall that by construction

$$
\boldsymbol{A}_{g, \ell}^{*}=\operatorname{Proj}\left(\bigoplus_{k} \Gamma\left(\overline{\boldsymbol{A}}_{g, \ell}, \bar{\omega}^{\otimes k}\right)\right),
$$

where $\overline{\boldsymbol{A}}_{g, \ell}$ is any arithmetic toroidal compactification and $\bar{\omega}$ its canonical semi-ample sheaf [14, Thm. 2.5 (3)]. The Koecher principle [14, Prop. 1.5 ii] asserts that the restriction morphism

$$
\Gamma\left(\overline{\boldsymbol{A}}_{g, \ell}, \bar{\omega}^{\otimes k}\right) \longrightarrow \Gamma\left(\boldsymbol{A}_{g, \ell}, \bar{\omega}^{\otimes k}\right)
$$

is an isomorphism. In the application of the Koecher principle we used the assumption $g \geq 2$. Finally, from the finite étale canonical projection morphism (3.6) we derive an integral inclusion of algebras

$$
\pi_{i}^{*}: \bigoplus_{k} \Gamma\left(\boldsymbol{A}_{g, \ell_{i}}[1 / \ell], \omega_{i}^{\otimes k}\right) \hookrightarrow \bigoplus_{k} \Gamma\left(\boldsymbol{A}_{g, \ell}, \omega^{\otimes k}\right) .
$$

This establishes the claim. Therefore, since $\widetilde{\boldsymbol{A}}_{g, \ell}^{(i)}$ is constructed from $\boldsymbol{A}_{g, \ell_{i}}^{*}$ by normalization, we obtain a chain of finite morphisms

$$
\widetilde{\boldsymbol{A}}_{g, \ell}^{(i)}[1 / \ell] \stackrel{p_{i}}{\rightarrow} \boldsymbol{A}_{g, \ell}^{*} \rightarrow \boldsymbol{A}_{g, \ell_{i}}^{*}[1 / \ell] .
$$


The morphism $p_{i}$ is birational, since $\widetilde{\boldsymbol{A}}_{g, \ell}^{(i)}[1 / \ell]$ and $\boldsymbol{A}_{g, \ell}^{*}$ both contain the open subset $\boldsymbol{A}_{g, \ell}$. Because $\boldsymbol{A}_{g, \ell}^{*}$ is known to be normal, we can invoke Zariski's main theorem to deduce that $p_{i_{*}} \mathcal{O}_{\widetilde{\boldsymbol{A}}_{g, \ell}^{(i)}[1 / \ell]}=\mathcal{O}_{\boldsymbol{A}_{g, \ell}^{*}}$. Because $p_{i}$ is finite, we finally infer that $p_{i}$ has to be an isomorphism. All in all, we can glue the schemes $\widetilde{\boldsymbol{A}}_{g, \ell}^{(i)}$ into a single scheme $\boldsymbol{A}_{g, \ell}^{*}$ over Spec $Z\left[\zeta_{\ell}\right]$ with the desired properties. A similar argument applies to the sheaves $\omega_{i}$ and $\widetilde{\omega}_{i}$, and shows that we can glue the $\widetilde{\omega}_{i}$ into a single sheaf $\omega$ as expected.

To finish the proof, we have to justify that properties $i$-iii characterize $\boldsymbol{A}_{g, \ell}^{*}$ and $\omega$. Let us show that any scheme $X$ fulfilling the same properties coincides with $\boldsymbol{A}_{g, \ell}^{*}$ as constructed above. The characterization of $\omega$ will then be straightforward. Such a scheme $X$ contains $\boldsymbol{A}_{g, \ell}^{*}[1 / \ell]$ as a dense open subset, by assumption. Furthermore, the open subset $X\left[1 / \ell_{i}\right]$ is finite over $\boldsymbol{A}_{g, \ell_{i}}^{*}\left[1 / \ell_{i}\right]$, extending the natural projection $\boldsymbol{A}_{g, \ell} \rightarrow \boldsymbol{A}_{g, \ell_{i}}[1 / \ell]$. By the very construction of $\boldsymbol{A}_{g, \ell}^{*}\left[1 / \ell_{i}\right]$ as a normalisation of $\boldsymbol{A}_{g, \ell_{i}}^{*}$, it follows there is a sequence of finite morphisms

$$
\boldsymbol{A}_{g, \ell}^{*}\left[1 / \ell_{i}\right] \stackrel{p}{\rightarrow} X\left[1 / \ell_{i}\right] \rightarrow \boldsymbol{A}_{g, \ell_{i}}^{*} .
$$

The first arrow $p$ is birational and finite, and $X\left[1 / \ell_{i}\right]$ is normal. By Zariski's main theorem $p$ has to be an isomorphism. Since this is true for $i=1,2$, we conclude that $X=\boldsymbol{A}_{g, \ell}^{*}$.

We now proceed to explain how to place ourselves in the situation of 3.1. For this, let us introduce a projective smooth arithmetic toroidal compactification $\overline{\boldsymbol{A}}_{g, \ell}$, defined over Spec Z $\left[\zeta_{\ell}, 1 / \ell\right]$. Such compactifcations exist as schemes by [14, Chap. V, Sec. 5]. There is a natural surjective morphism $\varphi: \overline{\boldsymbol{A}}_{g, \ell} \rightarrow \boldsymbol{A}_{g, \ell}^{*}$ over $Z\left[\zeta_{\ell}, 1 / \ell\right]$. By taking the closure of its graph in a projective space over $Z\left[\zeta_{\ell}\right]$, we see there exists a projective and flat scheme over Spec Z[ $\left[\zeta_{\ell}\right]$, still denoted $\overline{\boldsymbol{A}}_{g, \ell}$, and a proper morphism $\overline{\boldsymbol{A}}_{g, \ell} \rightarrow \boldsymbol{A}_{g, \ell}^{*}$ extending $\varphi$ to Spec $Z\left[\zeta_{\ell}\right]$. We will use the same notation for the extended morphism. Alternatively, by [14, Chap. V, Thm. 5.8], we know that, over $Z\left[\zeta_{\ell}, 1 / \ell\right], \overline{\boldsymbol{A}}_{g, \ell}$ can be obtained from $\boldsymbol{A}_{g, \ell}^{*}$ as the normalization of a blow up along a closed subscheme $T \subset \boldsymbol{A}_{g, \ell}^{*}$. Then one may take the schematic closure of $T$ in $\boldsymbol{A}_{g, \ell}^{*}$ over $Z\left[\zeta_{\ell}\right]$, and the normalization of its blow-up. We point out that the extended scheme $\overline{\boldsymbol{A}}_{g, \ell}$ may not be smooth over Spec Z $\left[\zeta_{\ell}\right]$, but this is not required in the constructions of 3.1 .

By [14, Chap. V, Thm. 2.5 and Sec. 4], the ample line bundle $\boldsymbol{\omega}$ on $\boldsymbol{A}_{g, \ell}^{*}$ carries a natural singular hermitian $L^{2}$ type metric, whose singularities are of logarithmic type along $F=\boldsymbol{A}_{g, \ell}^{*} \backslash \boldsymbol{A}_{g, \ell}$. By [25, Thm. 3.1], [7, Thm. 5.3], the resulting hermitian line bundle $\bar{\omega}$ is pre-log-log in the sense of Definition 3.1. We may thus consider the height function $h_{\bar{\omega}}$ obtained by the 
procedure of $\S 3.1$, with respect to $\varphi: \overline{\boldsymbol{A}}_{g, \ell} \rightarrow \boldsymbol{A}_{g, \ell}^{*}$. An important feature we will use is summarized in the following lemma.

LEMMA 3.10. Let $B$ be an abelian variety over a number field $L$, with a principal polarization and level $\ell$ structure. Let $z$ be the cycle it defines in $\boldsymbol{A}_{g, \ell}^{*}$, and let $h_{F}(B)$ denote Faltings canonical height of $B$. Then we have:

$$
h_{F}(B)=\widetilde{h}_{\bar{\omega}}(z)
$$

Proof. The proof is an adaptation of [14, p. 169], and we just sketch the main lines. To simplify the notations, we assume that the $\ell_{i}$ are prime. Observe that $B$ has semi-stable reduction over Spec $\mathcal{O}_{L}$, and has level $\ell_{i}$ structures too. One can define heights on $\boldsymbol{A}_{g, \ell_{i}}^{*}$ with respect to the natural hermitian line bundles $\bar{\omega}_{i}$. These heights however take values in $\mathbb{R} / Q \log \ell_{i}$, because the base rings are $Z\left[\zeta_{\ell_{i}}, 1 / \ell_{i}\right]$. As in $[14$, p. 169], one shows that

$$
h_{F}(B)=\widetilde{h}_{\bar{\omega}_{i}}\left(z_{i}\right) \quad \text { in } \frac{\mathbb{R}}{\mathrm{Q} \log \ell_{i}}
$$

where $z_{i}$ is the cycle defined by $B$ in $\boldsymbol{A}_{g, \ell_{i}}^{*}$. By functoriality properties of heights, one also has

$$
\widetilde{h}_{\bar{\omega}}(z)=\widetilde{h}_{\bar{\omega}_{i}}\left(z_{i}\right) \quad \text { in } \frac{\mathbb{R}}{\mathrm{Q} \log \ell_{i}} .
$$

Because $\ell_{1}$ and $\ell_{2}$ are relatively prime, equations (3.7)-(3.8) imply the equality of real numbers

$$
h_{F}(B)=\widetilde{h}_{\bar{\omega}}(z)
$$

as was to be shown.

Let us now briefly describe how we will apply the results in 3.2 . Let $\mathscr{f}$ be an arithmetic surface and $A$ a non-isotrivial ${ }^{10}$ abelian variety over $K(\mathscr{B})$. We assume that $A$ comes equipped with a level $\ell$ structure and a principal polarization. Then, $A$ has semi-stable reduction at codimension 1 points of $\mathscr{C}$. Therefore, it extends to a semi-abelian scheme $\mathscr{C} \rightarrow \mathscr{C}$, for some big open subset $\mathscr{C}$ of $\mathscr{C}$. Associated to $\mathscr{b}_{K} \rightarrow \mathscr{C}_{K}$ there is a clas-

$\left({ }^{10}\right)$ In our setting, by isotrivial we mean an abelian variety over $\mathscr{C}_{K}$ that has constant Néron model after a finite extension of $\mathscr{C}_{K}$. 
sifying map

$$
\gamma: \mathscr{C}_{K} \longrightarrow \boldsymbol{A}_{g, \ell}^{*}
$$

Observe that $\gamma$ is finite over its image, since we assumed that $A$ is nonisotrivial. Let us denote by $\mathscr{Z}$ the Zariski closure of the push-forward cycle $\gamma_{*} \mathscr{O}_{K}$. Observe that because $A$ is abelian, $\mathscr{Z}$ non-trivially intersects the open subscheme $\boldsymbol{A}_{g, \ell}$. Given a generic sequence of integral points $P_{n}$ : Spec $\mathcal{O}_{L_{n}} \rightarrow \mathscr{C} 6$, mapping the generic point into the good reduction locus, we obtain a generic sequence in $\mathscr{Z}(\bar{K}) \backslash F(\bar{K})$. Let us assume, for simplicity, that $\mathscr{Z}$ is reduced (latter we will consider the general case). Then by Theorem 3.5 and Lemma 3.10, we derive the inequality

$$
\widetilde{h}_{\bar{\omega}}(\mathscr{Z}) \leq \liminf _{n} h_{F}\left(P_{n}^{*} \mathscr{b}\right) .
$$

We may thus obtain bounds for the height of $\mathscr{Z}$ from bounds on the Faltings heights of the semi-abelian schemes $P_{n}^{*}, b$ over number fields. These considerations will actually be carried out for families of cycles provided by quotients of $A$ by the levels of $p$-divisible groups. A crucial step will be to choose suitable integral points so as to get uniform bounds for their Faltings heights.

REMARK 3.11. For a given sequence of integral points, the right hand side of (3.9) may actually be infinite. An example is given by the universal generalized elliptic curve over the regular modular curve $X(\ell)$, and the generic sequence of CM points. ${ }^{11}$ For this reason, to produce sequences for which the limit is finite, we will apply Autissier's effective version of Rumely's theorem, with control on the heights [2].

\section{Heights of cycles in moduli spaces of abelian varieties}

As an application of the results of the preceding sections, we show how to uniformly bound the heights of the cycles induced by the quotients of an abelian variety by the levels of a Barsotti-Tate subgroup, over the field of fractions of an arithmetic surface. The bounds we provide imply the finiteness of this family of abelian varieties, up to isomorphism. According to

(11) In particular, this ruins the hope of obtaining Duke's equidistribution of Heegner points [10] by Arakelov theoretic means, in the style of Szpiro-UllmoZhang [31]. 
Proposition 1.2, this gives another proof of Tate's conjecture for abelian varieties over finitely generated fields of transcendence degree 1 over $Q$. Actually, by Zarhin's trick (cf. Proposition 1.3) and a standard Galois descent argument, it will be enough to treat the case of principally polarized abelian varieties with a sufficiently high level structure.

Let $\ell$ be a positive integer which is a product of two relatively prime integers $\ell_{1}, \ell_{2} \geq 3$. Let $\mathscr{X} \rightarrow \operatorname{Spec} \mathcal{O}_{K}$ be an arithmetic surface and $A$ an abelian variety over $K(\mathscr{B})$, endowed with a level $\ell$ structure and a principal polarization. To exclude trivial cases, we assume that $A$ is not isotrivial. Fix a prime $p$ not dividing $\ell$, and let $\left\{G_{n}\right\}_{n}$ be a Barsotti-Tate subgroup of $A\left[p^{\infty}\right]$ defining a Lagrangian subspace of $T_{p} A \otimes_{z_{p}} Q_{p}$. The quotient abelian varieties $A / G_{n}$ are non-isotrivial and inherit level $\ell$ structures and principal polarizations. For every $n, A / G_{n}$ has semi-stable reduction at codimension 1 points of $\mathscr{X}$. They all extend to semi-abelian schemes over $\mathscr{C}_{K}$, with common bad reduction by the Serre-Tate theorem. We may consider the induced classifying maps $\gamma_{n}: \mathscr{X}_{K} \rightarrow \boldsymbol{A}_{g, \ell}^{*}$, which are finite onto their images by nonisotriviality. We denote by $\mathscr{E}_{n}$ the Zariski closure of the direct image cycles $\gamma_{n *} \cdot \mathscr{C}_{K}$. These are 2-dimensional effective cycles in $\boldsymbol{A}_{g, \ell}^{*}$, possibly with multiplicity, flat over Spec Z, which meet the open subscheme $\boldsymbol{A}_{g, \ell}$. In this section we derive uniform bounds for the degree and the height of the cycles $\mathscr{Z}_{n}$ with respect to $\bar{\omega}$, following the strategy outlined in 3.2.

We begin by bounding the multiplicity and degree of $\mathscr{E}_{n}$.

Lemma 4.1. The multiplicity of the cycle $\mathscr{Z}_{n}$ in $\boldsymbol{A}_{g, \ell}^{*}$ and its degree with respect to $\omega_{\mathrm{Q}\left(\zeta_{\ell}\right)}$ are bounded independently of $n$.

Proof. We denote by $\mathscr{A}_{n}$ (resp. $\mathscr{6}$ ) the semi-abelian scheme over $\mathscr{C}_{K}$ extending $A_{n}$ (resp. $A$ ), and $\omega_{\mathscr{C}_{n} / \mathscr{C}_{K}}$ (resp. $\omega_{\mathscr{C} / \mathscr{C}_{K}}$ ) the determinant of the dual of the relative Lie algebra of $\mathscr{C}_{n}$ over $\mathscr{C}_{K}$. By construction, we have

$$
\begin{aligned}
\operatorname{deg}_{\omega_{Q\left(\zeta_{\ell}\right)}} \mathscr{L}_{n} & =\operatorname{deg} \gamma_{n}^{*} \omega_{Q\left(\zeta_{\ell}\right)} \\
& =\operatorname{deg} \omega_{\mathscr{C}_{n} / \mathscr{C}_{K}} .
\end{aligned}
$$

To explain the last equality, we must remark that the morphism. $\mathscr{C}_{K} \rightarrow \boldsymbol{A}_{g, \ell}^{*}$ factors through $\overline{\boldsymbol{A}}_{g, \ell}$, and that there is a universal semi-abelian scheme over $\overline{\boldsymbol{A}}_{g, \ell, \mathrm{Q}\left(\zeta_{\ell}\right)}$. Hence, by uniqueness of the semi-abelian extension of $A_{n}$, it follows that the pull-back of the universal semi-abelian scheme to $\mathscr{X}_{K}$ is indeed isomorphic to $\mathscr{A}_{n}$. This justifies the equality. Now, because the natural morphism $\mathscr{b} \rightarrow \mathscr{b}_{n}$ is étale (we are in characteristic 0 ), we have

$$
\operatorname{deg} \omega_{\mathscr{L}_{n} / \mathscr{C}_{K}}=\operatorname{deg} \omega_{\mathscr{C} / \mathscr{C}_{K}} .
$$


But this is an equality of strictly positive integers. Indeed, the non-isotriviality assumption is equivalent to $\operatorname{deg} \omega_{\mathscr{b} / \mathscr{C}_{K}}>0$ [14, Chap. V, Prop. 2.2]. It follows from these observations that the multiplicity and degree of $\mathscr{Z}_{n}$ are bounded independently of $n$.

We now construct suitable sequences of integral points in the cycles $\mathscr{Z}_{n}$. Let $\mathscr{C}$ be a big open subset of $\mathscr{C}$ to which $A$ extends. Denote by $\mathscr{b} \rightarrow \mathscr{Q} b$ the extended semi-abelian scheme. Let $D$ be the divisor of bad reduction and $\mathscr{V}=\mathscr{C} \backslash D$. In principle, the Barsotti-Tate group $\left\{G_{n}\right\}_{n}$ might not extend to a Barsotti-Tate subgroup of $\mathscr{b} \mid \mathscr{V}$. However, by Proposition 2.1, this will be true after possibly shrinking $\mathscr{C}$ (into a big open subset) and replacing $A$ by $A / G_{m}$ and $G_{n}$ by $G_{n+m} / G_{m}$, for some $m \geq 0$. The effect of this on the family $\left\{\mathscr{Z}_{n}\right\}_{n}$ is just to exclude a finite number of cycles. Therefore we can suppose that $m=0$. We are now in the situation of 2.3 and Theorem 2.10. We employ the same notations. In particular, we have the finite subscheme $Y \subset \mathscr{C}$, the blow-up $\mathrm{Bl}_{Y}(\mathscr{C})$, the open subset $\mathscr{\mathscr { W }}$ of $\mathrm{Bl}_{Y}(\mathscr{C} 6)$ and the integer $n_{1}$ for which Theorem 2.10 holds.

The quotient $A / G_{n_{1}}$ extends to a semi-abelian scheme over a big open subset $\Omega$ of $\mathrm{Bl}_{Y}(\mathscr{B})$. After a finite sequence of blow-ups centered over $\mathrm{Bl}_{Y}(\mathscr{C}) \backslash \Omega$, we obtain an arithmetic surface $\widetilde{\mathscr{C}}$ over which $A / G_{n_{1}}$ extends to a semi-abelian scheme $\mathscr{B} \rightarrow \widetilde{\mathscr{X}}$. Observe that $\mathscr{\mathscr { W }} \cap \Omega$ naturally embeds as an open subset of $\widetilde{\mathscr{C}}$. Moreover, because $\Omega$ is big in $\mathrm{Bl}_{Y}(\mathscr{C})$, we still have that the projection $\mathscr{W} \cap \Omega \rightarrow \operatorname{Spec} \mathcal{O}_{K}$ is surjective.

Proposition 4.2. There exist a real constant $C$ and an infinite sequence of integral points $P: \operatorname{Spec} \mathcal{O}_{L} \rightarrow \mathscr{C}$ ( $L$ a varying extension of K) such that:

i. $P$ sends Spec $L$ into $\mathscr{T}_{K}$, and hence $P^{*} A / P^{*} G_{n}$ is defined;

ii. we have

$$
h_{F}\left(P^{*} A / P^{*} G_{n}\right)=h_{F}\left(P^{*} A / P^{*} G_{n_{1}}\right) \leq C
$$

for every $n \geq n_{1}$.

Proof. For every integral point $P$ : Spec $\mathcal{O}_{L} \rightarrow \mathscr{W}$, Theorem $2.10 \mathrm{ii}$ and Faltings' theorem as stated in [15, Chap. IV, Sec. 3, (3.1)] give

$$
h_{F}\left(P^{*} A / P^{*} G_{n}\right)=h_{F}\left(P^{*} A / P^{*} G_{n_{1}}\right), \quad n \geq n_{1} .
$$

Therefore, it is enough to produce an infinite sequence of integral points $P$ of $\mathscr{\mathscr { V }}$ for which $h_{F}\left(P^{*} A / P^{*} G_{n_{1}}\right)$ is bounded independently of $P$. 
We apply Autissier's effective version of Rumely's theorem [2, Thm. 4.2.3]. To fulfill the hypothesis of loc. cit., we need an arithmetic surface of generic genus $\geq 1$ and a good height. We satisfy these requirements by fixing the following data:

- a covering $\pi: \widetilde{\mathscr{C}}_{1} \rightarrow \widetilde{\mathscr{X}}$, where $\widetilde{\mathscr{C}}_{1}$ is an arithmetic surface of generic genus $\geq 1$, defined over Spec $\mathcal{O}_{K^{\prime}}, K \subset K^{\prime}$. We write $\mathscr{B}_{1}=\pi^{*} \mathscr{B}$.

- A continuous hermitian metric $\|\cdot\|_{0}$ over $\omega_{\mathscr{B}_{1} / \widetilde{\mathscr{O}}_{1}}$, with semi-positive first Chern current and with $\|\cdot\|_{0} \leq\|\cdot\|$. Here $\|\cdot\|$ is the natural pre-log$\log L^{2}$ metric. The metric $\|\cdot\|_{0}$ can be build from $\|\cdot\|$ by truncation and rescaling, as in the proof of Theorem 3.5.

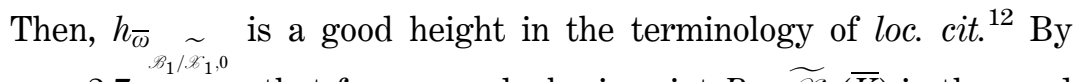
Lemma 3.7, we see that for every algebraic point $P \in \widetilde{\mathscr{C}}_{1}(\bar{K})$ in the good reduction locus of $\mathscr{B} 1$, we have

$$
\begin{aligned}
& h_{F}\left((\pi \circ P)^{*} \mathscr{B}\right)=h_{F}\left(P^{*} \mathscr{B}_{1}\right) \\
& =\widetilde{h}_{\bar{\omega}} \underset{\mathscr{S}_{1} / \mathscr{\mathscr { C }}_{1}}{ }(P) \\
& \leq \widetilde{h}_{\bar{\omega}} \underset{\mathscr{B}_{1} / \mathscr{D}_{1}, 0}{ }(P) .
\end{aligned}
$$

Introduce $\Omega_{1}=\pi^{-1}(\Omega \cap \mathscr{W}) \subseteq \widetilde{\mathscr{C}}_{1}$. This is an open subset surjecting to Spec $\mathcal{O}_{K^{\prime}}$. We recursively apply Autissier's theorem, starting with $\Omega_{1}$ and taking as height $h_{\bar{\omega}} \sim$. This produces an infinite sequence of integral points $P: \operatorname{Spec} \mathcal{O}_{L} \rightarrow \Omega_{1}\left(L\right.$ a varying extension of $\left.K^{\prime}\right)$ such that

$$
\widetilde{h}_{\bar{\omega}} \underset{\mathscr{S}_{1} / \mathscr{\mathscr { X }}_{1}, 0}{ }(P) \leq C,
$$

for some real constant $C$ that only depends on the hermitian line bundle $\bar{\omega}_{\mathscr{B}_{1} / \widetilde{\mathscr{X}}_{1,0}}$ and the singular fibers of $\widetilde{\mathscr{C}}_{1}$. Projecting these integral points back to $\Omega \cap \mathscr{W}$ and taking into account (4.1), we obtain the desired infinite sequence of integral points.

THEOREM 4.3. The family of abelian varieties up to isomorphism $\left\{A_{n}\right\}_{n} / \simeq$ is finite.

${ }^{12}$ ) The required positivity of $\omega_{\mathscr{R}_{1} / \widetilde{\mathscr{X}}_{1}}$ on vertical curves is ensured by [14, Chap. V, Prop. 2.2]. 
Proof. First we establish the finiteness of the cycles $\mathscr{L}_{n}$. Fix a family of integral points $\left\{P_{k}\right\}_{k}$ as in proposition 4.2. This family induces a family of integral points in $\mathscr{D}_{n}$, that we denote by $\left\{P_{k}^{(n)}\right\}_{k}$. The non-isotriviality assumption ensures that this sequence is infinite. By Theorem 3.5, Lemma 3.10 and Proposition 4.2, we obtain for $n \geq n_{1}$

$$
\begin{aligned}
\widetilde{h}_{\overline{\boldsymbol{\omega}}}\left(\mathscr{D}_{n, \text { red }}\right) & \leq 2 \liminf _{k} \widetilde{h}_{\bar{\omega}}\left(P_{k}^{(n)}\right) \\
& =2 \liminf _{k} h_{F}\left(P_{k}^{*} A / P_{k}^{*} G_{n}\right) \\
& =2 \liminf _{k} h_{F}\left(P_{k}^{*} A / P_{k}^{*} G_{n_{1}}\right) \leq C .
\end{aligned}
$$

Moreover we proved that the multiplicities of the cycles $\mathscr{Z}_{n}$ are bounded (Lemma 4.1). Therefore the heights $h_{\bar{\omega}}\left(\mathscr{Z}_{n}\right)$ are bounded. Because the degrees of the $\mathscr{Z}_{n}$ with respect to $\omega_{Q\left(\zeta_{\ell}\right)}$ are bounded as well, by Corollary 3.4 we derive the finiteness of the family $\left\{\mathscr{Z}_{n}\right\}_{n}$. Unfortunately this is not enough to conclude, for we have to show that the morphisms $\gamma_{n}: \mathscr{C}_{K} \rightarrow \boldsymbol{A}_{n, \ell}^{*}$ arise in a finite family.

For every $n$, let us define $\Gamma_{n}$ as the closure of the graph of $\gamma_{n}$ in $\mathscr{O} \times \boldsymbol{A}_{n, \ell}^{*}$. It will be enough to bound the degree and height of the cycles $\Gamma_{n}$ with respect to an ample pre-log-log hermitian line bundle on $\mathscr{C} \times \boldsymbol{A}_{n, \ell}^{*}$.

We introduce $\overline{\mathcal{O}\left(P_{1}\right)}$ on $\mathscr{C}$, whose underlying line bundle is attached to the integral point $P_{1}$ as above and whose metric is fixed, smooth and with positive first Chern form. We take $\overline{\mathscr{C}}=p_{1}^{*} \overline{\mathcal{O}\left(P_{1}\right)} \otimes p_{2}^{*} \bar{\omega}$ on $\mathscr{O} \times \boldsymbol{A}_{n, \ell}^{*}$. Because $\mathcal{O}\left(P_{1}\right)$ is only known to be generically ample, $\overline{\mathscr{C}}$ does not strictly fulfill the requirements of Corollary 3.4. However this may be solved after a change of model, as in [5, Prop. 3.2.2 (ii)]. This will only alter the height by a quantity controlled by the degree of $\mathscr{Z}_{n}$ with respect to $\omega_{\mathrm{Q}\left(\zeta_{\ell}\right)}$, hence bounded. We leave to the reader the task of writing down these details.

The degree of $\Gamma_{n}$ with respect to $\mathscr{L}_{K}$ is the degree of $\mathscr{Z}_{n}$ with respect to $\omega_{Q\left(\zeta_{\ell}\right)}$, hence bounded. For the height, we compute according to the definition and applying the projection formula. We find

$$
\begin{aligned}
h_{\overline{\mathscr{L}}}\left(\Gamma_{n}\right)= & \widehat{\operatorname{deg}}\left(\Gamma_{n} \mid \hat{\mathrm{c}}_{1}(\overline{\mathscr{D}})^{2}\right) \\
& =h_{\overline{\mathcal{O}\left(P_{1}\right)}}(\mathscr{C})+h_{\overline{\boldsymbol{\omega}}}\left(\mathscr{\mathscr { C }}_{n}\right)+2 \widehat{\operatorname{deg}}\left(\Gamma_{n} \mid \hat{\mathrm{c}}_{1}\left(p_{1}^{*} \overline{\mathcal{O}\left(P_{1}\right)}\right) \hat{\mathrm{c}}_{1}\left(p_{2}^{*} \bar{\omega}\right)\right) .
\end{aligned}
$$

The first term does not depend on $n$, while the second one is bounded by the finiteness of the family $\left\{\mathscr{Z}_{n}\right\}_{n}$. For the last term, the definition of height 
pairing gives

$$
\begin{aligned}
& \widehat{\operatorname{deg}}\left(\Gamma_{n} \mid \hat{\mathrm{c}}_{1}\left(p_{1}^{*} \overline{\mathcal{O}\left(P_{1}\right)}\right) \hat{\mathrm{c}}_{1}\left(p_{2}^{*} \overline{\boldsymbol{\omega}}\right)\right)= \\
& {\left[K\left(P_{1}\right): \mathrm{Q}\right] h_{F}\left(P_{1}^{*} A / P_{1}^{*} G_{n}\right)-\int_{\Gamma_{n}(\mathrm{C})} p_{1}^{*}(\log \|\mathbf{1}\|) \mathrm{c}_{1}\left(p_{2}^{*} \overline{\boldsymbol{\omega}}\right) .}
\end{aligned}
$$

Here 1 is the canonical rational section of $\mathcal{O}\left(P_{1}\right)$. The Faltings height of $P_{1}^{*} A / P_{1}^{*} G_{n}$ is bounded by $C$ for $n \geq n_{1}$. For the archimedian factor, we recall that because $\mathscr{b} \rightarrow \mathscr{b}_{n}$ is étale, we have $\omega_{\mathscr{b}_{n} / \mathscr{C}_{K}} \simeq \omega_{\mathscr{b} \mid \mathscr{C}_{K}}$. At the level of metrics, this isomorphism is an isometry up to a constant (given by the rank of $G_{n}$ ). Therefore, the first Chern differential forms satisfy

$$
\begin{aligned}
p_{1 *} \mathrm{c}_{1}\left(p_{2}^{*} \bar{\omega}_{\mathrm{C}}\right) & =\mathrm{c}_{1}\left(\overline{\boldsymbol{\omega}}_{\mathscr{C}_{n} / \mathscr{O}_{K}, \mathrm{C}}\right) \\
& =\mathrm{c}_{1}\left(\overline{\boldsymbol{\omega}}_{\mathscr{C} / \mathscr{C}_{K}, \mathrm{C}}\right)
\end{aligned}
$$

and we conclude

$$
\int_{\Gamma_{n}(\mathrm{C})} p_{1}^{*}(\log \|\mathbf{1}\|) \mathrm{c}_{1}\left(p_{2}^{*} \overline{\boldsymbol{\omega}}\right)=\int_{\mathscr{C}(\mathrm{C})} \log \|\mathbf{1}\| \mathrm{c}_{1}\left(\overline{\boldsymbol{\omega}}_{\mathscr{C} / \mathscr{X}_{K}, \mathrm{C}}\right),
$$

which does not depend on $n$. This completes the proof of the theorem.

Remark 4.4. $i$. The constant $C$ in Proposition 4.2, and involved in the proof of Theorem 4.3, can be made explicit. For this, one has to combine other variants of Rumely's theorem, as for instance in [2, Sec. 4.6], and universal lower bounds for Faltings heights of abelian varieties over number fields (in unpublished work of the first author, see also [3]).

$i i$. It would be interesting to show that the heights $\widetilde{h}_{\bar{\omega}}\left(\mathscr{Z}_{n}\right)$ stabilize for $n$ big enough, by working directly on the definition of height.

Acknowledgments. It is a pleasure to thank E. Goren for suggesting to apply finiteness results on heights of cycles defined by logarithmically singular hermitian line bundles to families of abelian varieties, and $J$. Kramer for helpful remarks. We are grateful to the referee for useful comments on a first version of this article. Finally we warmly thank R. Bost for his help in the preparation of the figure. 


\section{REFERENCES}

[1] A. AbBes, Hauteurs et discrétude, Sém. Bourbaki, Exposé 825, Astérisque 245, pp. 141-166.

[2] P. Autissier, Points entiers sur les surfaces arithmétiques, J. Reine Angew. Math. 531 (2001), pp. 201-235.

[3] P. Autissier, Hauteur de Faltings et hauteur de Néron-Tate du diviseur thêta, Compos. Math. 142 (2006), pp. 1451-1458.

[4] J.-B. Bost, Letter to Ulf Kühn.

[5] J.-B. Bost - H. GILlet - C. SoulÉ, Heights of projective varieties and positive Green forms, J. Amer. Math. Soc. 7 (1994), pp. 903-1027.

[6] J. I. Burgos - J. Kramer - U. KüHn, Cohomological arithmetic Chow rings, J. Inst. Math. Jussieu 6 (2007), pp. 1-172.

[7] J. I. Burgos - J. Kramer - U. Kühn, Arithmetic characteristic classes of automorphic vector bundles, Documenta Math. 10 (2005), pp. 619-716.

[8] P. Deligne, Théorie de Hodge. II, Institut des Hautes Études Scientifiques. Publications Mathématiques, 40 (1971), pp. 5-57.

[9] P. Deligne - M. RApoport, Les schémas de modules de courbes elliptiques, Lecture Notes in Math. 349 (Springer, 1973), pp. 143-316.

[10] W. Duke, Hyperbolic distribution problems and half-integral weight Maass forms, Invent. Math. 92 (1988), pp. 73-90.

[11] G. Faltings, Arakelov's theorem for abelian varieties, Invent. Math. 73 (1983), pp. 337-347.

[12] G. FALTINGS, Endlichkeitssätze für abelsche Varietäten über Zahlkörpern, Invent. Math. 73 (1983), pp. 349-366.

[13] G. Faltings, Finiteness theorems for abelian varieties over number fields, Arithmetic Geometry (G. Cornell and J. H. Silverman, eds.), Springer-Verlag, 1986, pp. 9-27.

[14] G. FaltingS - C.-L. Chai, Degeneration of abelian varieties, Springer-Verlag, Berlin, 1990.

[15] G. Faltings - G. Wüstholz et al., Rational points, Aspects of Mathematics, Vol. E6, Vieweg, 1984.

[16] G. FreiXas I MontPlet, Heights and metrics with logarithmic singularities, J. Reine Angew. Math. 627 (2009), pp. 97-153.

[17] A. GRothendeck, Technique de descente et théorèmes d'existence en géométrie algébrique, III, Sém. Bourbaki, Exposé 212, 1961.

[18] A. J. DE JoNG - F. OORT, On extending families of curves, J. Alg. Geom. 6 (1997), pp. 545-562.

[19] H. Ikoma, The Faltings-Moriwaki modular height and isogenies of elliptic curves, J. Math. Kyoto. Univ. 48 (2008), pp. 661-682.

[20] J. Jost - K. Zuo, Arakelov type inequalities for Hodge bundles over algebraic varieties, Part I: Hodge bundles over algebraic curves, J. Alg. Geom. 11 (2002), pp. 535-546.

[21] V. Maillot, Géométrie d'Arakelov des variétés toriques et fibrés en droites intégrables, Mém. Soc. Math. France, 80, SMF (2000).

[22] L. Moret-Bailly, Pinceaux de variétés abéliennes, Astérisque 129 (1985).

[23] L. Moret-Bailly, Groupes de Picard et problèmes de Skolem. I, Ann. Scient. Éc. Norm. Sup. 22 (1989), pp. 161-179 
[24] A. MoRIWAKI, The modular height of an abelian variety and its finiteness property, Adv. Stud. Pure Math. 45 (2006), pp. 157-187.

[25] D. Mumford, Hirzebruch's Proportionality Theorem in the Non-Compact Case, Invent. Math. 42 (1977), pp. 239-272.

[26] H. Randriambololona, Métriques de sous-quotient et théorème de HilbertSamuel arithmétique pour les faisceaux cohérents, J. Reine angew. Math. 590 (2006), pp. 67-88.

[27] M. RAYNAUD, Faisceaux amples sur les schémas en groupes et les espaces homogènes, Lecture Notes in Mathematics 119, Springer-Verlag, 1970.

[28] I. Reiner, Maximal Orders, London Math. Soc. Monograph New Series, Oxford University Press, New York, 2003.

[29] R. Rumely, Arithmetic over the ring of all algebraic integers, J. Reine. angew. Math. 368 (1986), pp. 127-133.

[30] L. SzPIRo, Séminaire sur les pinceaux arithmétiques: la conjecture de Mordell, Astérisque 127 (1985).

[31] L. SzPIRo - E. Ullmo - S. Zhang, Equirépartition des petits points, Invent. Math. 127 (1997), pp. 337-347.

[32] J. TATE, Endomorphisms of Abelian Varieties over Finite Fields, Invent. Math. 2 (1966), pp. 134-144.

[33] J. TATE, p-divisible groups, in Proceedings of a Conference on Local Fields, Driebergen, Springer-Verlag, 1967.

[34] Y. G. ZARHIN, Abelian varieties in characteristic p, Math. Notes of the Academy of Sciences of the USSR, 19 (1976), pp. 240-244.

[35] Y. G. ZARHIN, Endomorphisms of Abelian varieties over fields of finite characteristic, Math. USSR Izvestija, 9, No. 2 (1975), pp. 255-260.

[36] S. Zhang, Positive line bundles on arithmetic varieties, J. Amer. Math. Soc. 8 (1995), pp. 187-221.

Manoscritto pervenuto in redazione il 2 Dicembre 2011. 
